\title{
Probabilistic models of the Jovian magnetopause and bow shock locations
}

\author{
S. P. Joy, ${ }^{1}$ M. G. Kivelson, ${ }^{1,2}$ R. J. Walker, ${ }^{1,2}$ K. K. Khurana, ${ }^{1}$ C. T. Russell, ${ }^{1,2}$ \\ and T. Ogino ${ }^{3}$
}

Received 29 October 2001; revised 2 February 2002; accepted 7 February 2002; published 18 October 2002.

[1] New three-dimensional models of the Jovian magnetopause and bow shock were derived by combining spacecraft observations with boundary characteristics inferred from a magnetohydrodynamic (MHD) simulation. The MHD simulation provides polynomial forms parameterized by solar wind dynamic pressure. Observations from Pioneer 10 and 11, Voyager 1 and 2, Ulysses, and Galileo were used to establish the probability that regions surrounding Jupiter fall inside or outside these boundary surfaces. The magnetopause location was found to have a bimodal probability distribution with the two most probable standoff distances at $63 \mathrm{R}_{\mathrm{J}}\left(\sigma=4 \mathrm{R}_{\mathrm{J}}\right)$ and $92 \mathrm{R}_{\mathrm{J}}\left(\sigma=6 \mathrm{R}_{\mathrm{J}}\right)$. The bow shock location distribution appears bimodal, but a single distribution function description cannot be ruled out at the $95 \%$ confidence level. The mean bow shock standoff distance is $84 \mathrm{R}_{\mathrm{J}}\left(\sigma=16 \mathrm{R}_{\mathrm{J}}\right)$. Analysis of solar wind measurements near 5.2 AU (interplanetary magnetic field, dynamic pressure, and Alfvén Mach number) suggests that the bimodal distribution of boundary positions results at least in part from the bimodal distribution of solar wind parameters in the vicinity of Jupiter. The probability density distributions of these parameters within and between regions of disturbed solar wind, caused by corotating interaction regions and coronal mass ejections, are statistically distinct. Smaller variations were also observed in these parameters over the solar cycle. INDEX TERMS: 2724 Magnetospheric Physics: Magnetopause, cusp, and boundary layers; 2154 Interplanetary Physics: Planetary bow shocks; 7843 Space Plasma Physics: Numerical simulation studies; 6220 Planetology, Solar System Objects: Jupiter; 5737 Planetology, Fluid Planets: Magnetospheres (2756); KEYWORDS: Jupiter, bow shock, magnetopause, solar wind, MHD

Citation: Joy, S. P., M. G. Kivelson, R. J. Walker, K. K. Khurana, C. T. Russell, and T. Ogino, Probabilistic models of the Jovian magnetopause and bow shock locations, J. Geophys. Res., 107(A10), 1309, doi:10.1029/2001JA009146, 2002.

\section{Introduction}

[2] The solar wind is heated and deflected around Jupiter by the bow shock that forms upstream of the planet in the flow. The solar wind magnetosonic Mach number, plasma beta, and interplanetary magnetic field orientation determine the strength of the shock and influence its location [Farris and Russell, 1994]. As in all systems where shocks form, the shape of the shock is dependent on the shape of the obstacle [Spreiter et al., 1966; Stahara et al., 1989]. The bow shock diverts the solar wind around Jupiter's large magnetospheric cavity whose size and shape is determined by pressure balance at the magnetopause. At Jupiter, all internal pressure components (magnetic, thermal plasma pressure, and dynamic pressure) are important [Huddleston

\footnotetext{
${ }^{1}$ Institute of Geophysics and Planetary Physics, University of California, Los Angeles, CA, USA.

${ }^{2}$ Department of Earth and Space Sciences, University of California, Los Angeles, CA, USA.

${ }^{3}$ Solar Terrestrial Environment Laboratory, Nagoya University, Toyokawa, Aichi, Japan.

Copyright 2002 by the American Geophysical Union. 0148-0227/02/2001JA009146\$09.00
}

et al., 1998]. Due to the rapid rotation of Jupiter, and the large scale size of the system, centrifugal forces on the corotating plasma dominate over gravitational forces [Hill and Dessler, 1974]. The field configuration is stretched and disk-like [Engel and Beard, 1980], and the shock stands much closer to the magnetopause [Slavin et al., 1985; Stahara et al., 1989]. The solar wind dynamic pressure at 5.2 AU is highly variable, and consequently the Jovian magnetopause and bow shock shapes and locations are quite variable [Smith et al., 1978, 1981; Slavin et al., 1985].

[3] Seven spacecraft (Pioneer 10 and 11, Voyager 1 and 2, Ulysses, Galileo, and Cassini) have encountered the Jovian system. Galileo has been in orbit about Jupiter since December 1995. Cassini passed by Jupiter in December 2000 but data from that encounter are not yet available publicly. Figure 1 shows the trajectories of the spacecraft, and models of the magnetopause and bow shock based on the Voyager encounters [Lepping et al., 1981a]. The trajectories have been shaded to indicate whether the spacecraft was in the solar wind (black), the magnetosheath (light gray), or the magnetosphere (dark gray). Shading changes occur at the boundary crossings. Note that the boundaries have been observed over a wide range of local times and distances. The observed range of subsolar magnetopause positions is roughly between 50 


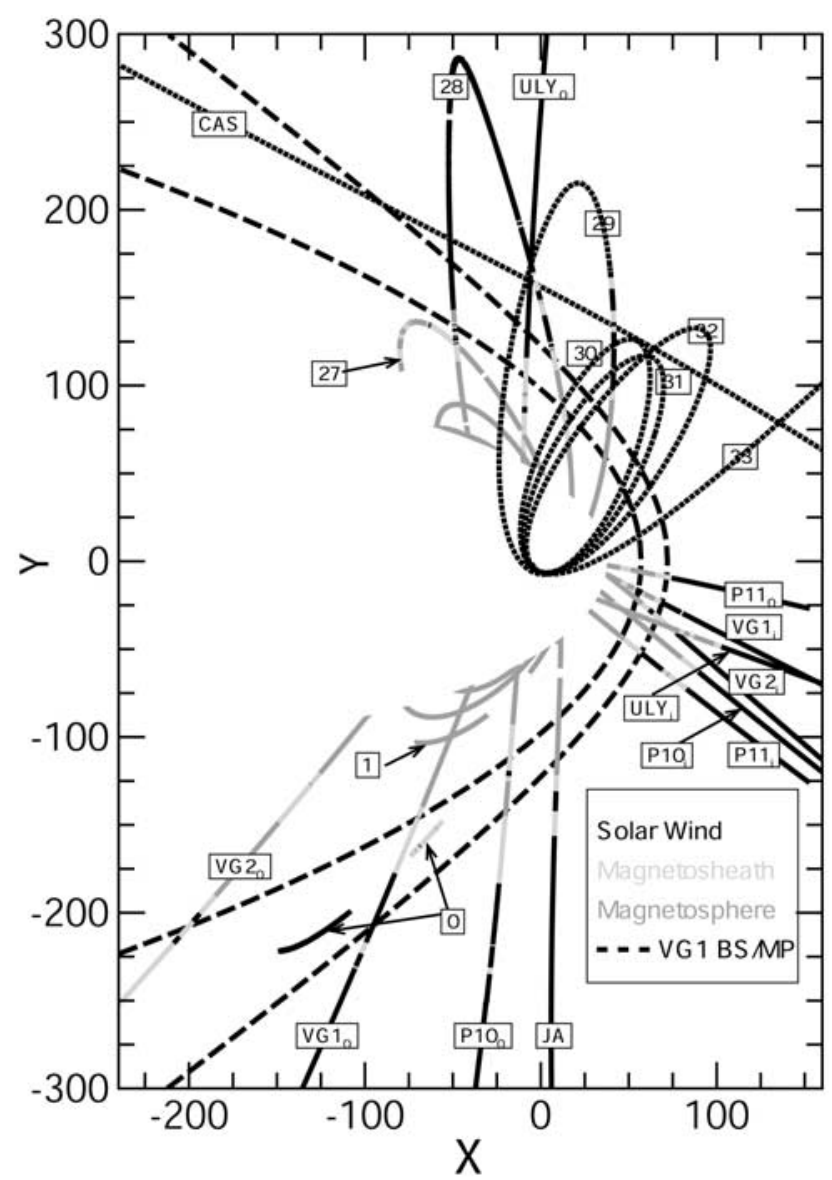

Figure 1. Summary of Jupiter observations to date. Trajectory data are shaded dark gray to indicate when the spacecraft was in the outer magnetosphere, light gray when in the magnetosheath, and black when in the solar wind, and are dotted when the data are not used in this study (Cassini, Galileo after Feb 1, 2001). Individual spacecraft trajectories are labeled by using subscripts on the inbound (i) and outbound (o) portions of the trajectories. Galileo orbits crossing boundary surfaces are labeled by using orbit number $(\mathrm{JA}=$ Jupiter Approach, $0=$ orbit 0, etc.). Except for Ulysses and Pioneer 11 outbound passes, all trajectories are near the Jupiter equatorial plane.

and 100 Jovian radii $\left(\mathrm{R}_{\mathrm{J}}=71492 \mathrm{~km}\right)$ and the range of subsolar bow shock positions are 55 to $125 \mathrm{R}_{\mathrm{J}}$.

[4] Previous empirical models of the boundary shapes and locations [Lepping et al., 1981a; Slavin et al., 1985; Huddleston et al., 1998] were based on least squares fits to an assumed conic section form of observed boundary crossings. Conic forms are inherently symmetric but there is likely to be an observable dawn-dusk asymmetry and polar flattening. The lack of low latitude dusk side data, and very limited high latitude data, has made it difficult to constrain model parameters that could represent asymmetry. Previous models of the Jovian boundary locations have either corrected for solar wind dynamic pressure effects [Slavin et al., 1985] or have allowed the boundary shapes and locations to vary in response to these changes [Huddleston et al., 1998]. Slavin et al. [1985] mapped crossings to their equivalent sub-solar point, using the shape model determined from the uncorrected crossings, and then corrected the values to a common pressure by using a model of the pressure dependence of the standoff distance. Their model is symmetric about the aberrated Sun-Jupiter line and assumes a circular cross-section about this line. Huddleston et al. [1998] provided evidence of polar flattening in the magnetopause by fitting mid and low latitude observations separately. In addition, they separated the Voyager and Pioneer crossing observations into high and low solar wind pressure events by using solar wind data from the other spacecraft of the pair, and correcting the data timing time to account for the spacecraft separation. Their analysis suggests that at high pressure, both the standoff distance and the flaring of the magnetopause and the bow shock are reduced relative to the low pressure case.

[5] In the model that we describe here, the response of the boundary shapes to different solar wind dynamic pressures was determined from runs of the Ogino-Walker magnetohydrodynamic (MHD) simulation of the Jovian magnetosphere [Ogino et al., 1998]. The shape models were then extended to a continuous family of surfaces by expressing the coefficients as functions of dynamic pressure. The most probable magnetopause and bow shock surfaces were then determined by relating the observations in the vicinity of Jupiter to the modeled forms of the surfaces.

\section{Ogino-Walker MHD Simulation at Jupiter}

[6] The Jupiter system MHD simulation of Ogino and Walker includes a spin axis aligned dipole magnetic field and a plasma source inside the inner boundary at $15 \mathrm{R}_{\mathrm{J}}$. Results have been generated for the case where the solar wind flow is orthogonal to the internal dipole [Ogino et al., 1998, Walker et al., 2001]. The simulation box is a halfspace with north-south symmetry that is elongated in the solar wind flow direction. This simulation has been used to investigate the impact of Jupiter's rapid rotation on the magnetospheric configuration, the magnetospheric response to changing interplanetary magnetic field (IMF) direction and strength at fixed solar wind dynamic pressure, and to changing solar wind dynamic pressure conditions in the absence of an IMF [Walker et al., 2001]. The simulation was run without an IMF until a steady state was achieved for solar wind dynamic pressures of $0.045,0.090,0.180$, and $0.360 \mathrm{nPa}$. These pressures were selected by scaling the nominal solar wind dynamic pressure at Jupiter, $\sim 0.092 \mathrm{nPa}$ [Slavin et al., 1985], by factors of two. However, these values correspond quite nicely with the mode (.04-.05 $\mathrm{nPa})$, mean (.099 $\mathrm{nPa})$, and upper quartile (.017-.018 $\mathrm{nPa}$ ) of the distribution of dynamic pressures observed by Pioneer 10 when it was in the solar wind near Jupiter [Slavin et al., 1985]. It should be noted that when the IMF vanishes, the MHD solution approaches the gas dynamic limit (infinite Alfvén Mach number) previously discussed [Slavin et al., 1985; Spreiter and Stahara, 1985] in the upstream region. Another set of results was generated at constant solar wind dynamic pressure $(0.090 \mathrm{nPa})$ by letting the IMF $(\mathrm{Bz})$ strength vary $( \pm 0.42 \mathrm{nT}$ and $\pm 0.84 \mathrm{nT})$.

[7] The bow shock is readily identifiable in all of the derived parameters of the Ogino-Walker simulation. For our purposes, the shock was defined to be the maximum in the thermal pressure gradient in the direction of solar wind flow 

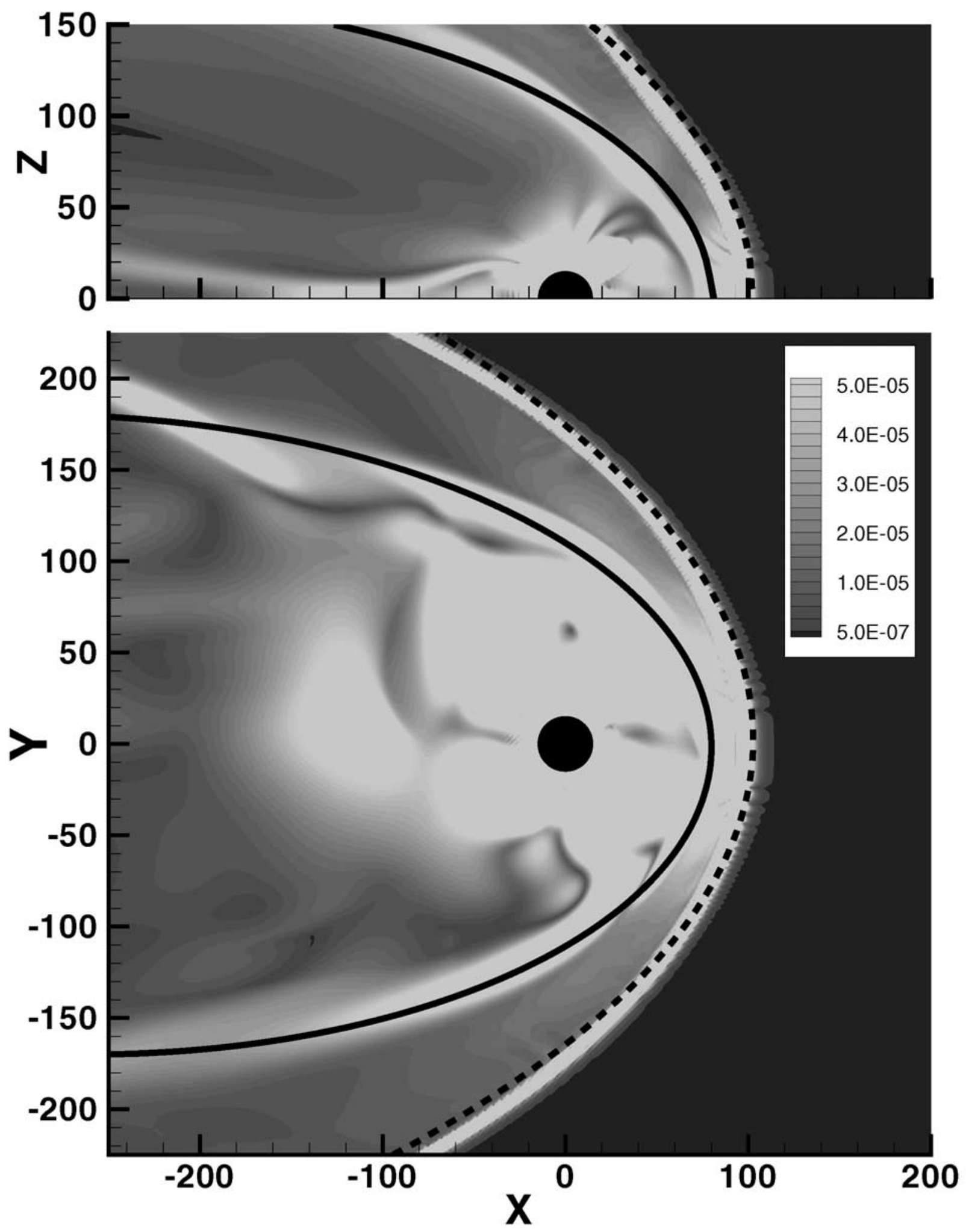

Figure 2. Model bow shock (dashed line) and magnetopause (solid line) locations calculated at a solar wind dynamic pressure of $0.090 \mathrm{nPa}$ are superimposed on contours of the gradient in the flow velocity magnitude from simulation results at the same pressure. Equatorial (bottom) and noon-midnight (top) planes are shown. 
Table 1. Summary of the Magnetopause and Bow Shock Shape Characteristics for the Various Ogino-Walker Simulation Results ${ }^{\mathrm{a}}$

\begin{tabular}{|c|c|c|c|c|c|c|c|c|c|c|c|c|c|c|}
\hline \multirow[t]{2}{*}{$\mathrm{P}_{\mathrm{d}}, \mathrm{nPa}$} & \multirow[t]{2}{*}{$\mathrm{B}_{\mathrm{z}}, \mathrm{nT}$} & \multicolumn{5}{|c|}{ Magnetopause } & \multicolumn{5}{|c|}{ Bow Shock } & \multicolumn{3}{|c|}{$\begin{array}{l}\text { Bow Shock to Magnetopause } \\
\text { Size Ratios } \\
\end{array}$} \\
\hline & & $\mathrm{X}$ & $Y_{\text {avg }}$ & $\mathrm{Z}$ & $\mathrm{Z} / \mathrm{Y}_{\mathrm{avg}}$ & $\begin{array}{c}\mathrm{Y}_{\text {dawn }} l \\
\mathrm{Y}_{\text {dusk }} \\
\end{array}$ & $\mathrm{X}$ & $\mathrm{Y}_{\mathrm{avg}}$ & $\mathrm{Z}$ & $\mathrm{Z} / \mathrm{Y}_{\mathrm{avg}}$ & $\begin{array}{c}\mathrm{Y}_{\text {dawn }} / \\
\mathrm{Y}_{\text {dusk }}\end{array}$ & $\mathrm{X}_{\mathrm{b}} / \mathrm{X}_{\mathrm{m}}$ & $\mathrm{Y}_{\mathrm{b}} / \mathrm{Y}_{\mathrm{m}}$ & $Z_{b} / Z_{m}$ \\
\hline 0.045 & 0 & 90 & 136 & 125 & 0.919 & 0.985 & 118 & 207 & 204 & 0.986 & 1.029 & 1.311 & 1.522 & 1.632 \\
\hline 0.090 & 0 & 76 & 112 & 107 & 0.951 & 0.940 & 100 & 174 & 173 & 0.994 & 1.012 & 1.316 & 1.553 & 1.617 \\
\hline 0.180 & 0 & 67 & 92 & 92 & 1.000 & 0.906 & 84 & 146 & 145 & 0.990 & 1.007 & 1.254 & 1.587 & 1.576 \\
\hline 0.360 & 0 & 58 & 78 & 80 & 1.026 & 0.867 & 72 & 124 & 123 & 0.988 & 0.992 & 1.241 & 1.590 & 1.538 \\
\hline 0.090 & 0.42 & 90 & 137 & 119 & 0.869 & 0.971 & 115 & 183 & 192 & 1.049 & 1.011 & 1.278 & 1.336 & 1.613 \\
\hline 0.090 & -0.42 & 94 & 146 & 125 & 0.856 & 0.940 & 142 & - & 200 & - & - & 1.511 & - & - \\
\hline 0.090 & 0.84 & 87 & 110 & 117 & 1.064 & 1.009 & 106 & 189 & - & - & 0.909 & 1.218 & 1.712 & - \\
\hline 0.090 & -0.84 & 95 & 122 & 123 & 1.008 & 1.017 & 151 & - & 223 & - & - & 1.589 & - & - \\
\hline
\end{tabular}

${ }^{\mathrm{a}}$ The solar wind dynamic pressure $\left(\mathrm{P}_{\mathrm{d}}\right)$ and $\mathrm{B}_{\mathrm{Z}}$ (IMF - pure north-south) columns are simulation parameters. A Jovicentric coordinate system is used where $\mathrm{X}$ points toward the Sun and $\mathrm{Z}$ is along the Jupiter spin axis. The $\mathrm{X}, \mathrm{Y}, \mathrm{Z}$ columns give the boundary locations along the respective axes in $\mathrm{R}_{\mathrm{J}}$. The ratios $\mathrm{Z} / \mathrm{Y}_{\text {avg }}$ and $\mathrm{Y}_{\text {dawn }} / \mathrm{Y}_{\text {dusk }}$ provide measures of polar flattening and dawn-dusk asymmetries, respectively. The bow shock to magnetopause size ratios show how the magnetosheath shape changes in response to variations in pressure and IMF strength and orientation. Missing values in the table indicate that the boundary was beyond the edge of the simulation box at the point of interest. The $\mathrm{P}_{d}=0.090 \mathrm{nPa}$ and $\mathrm{B}_{\mathrm{z}}=0.0 \mathrm{nT}$ row is shown in bold to facilitate comparison with runs at the same $\mathrm{P}_{\mathrm{d}}$ and finite $\mathrm{B}_{\mathrm{z}}$. This reveals the effect of a nonzero IMF on the shape of the boundaries.

$\left(\delta P_{t h} / \delta x\right)_{\max }$. Identifying the magnetopause surface in the simulation in an automated fashion is not straightforward. Several criteria were evaluated and rejected prior to settling for a criterion based on flow diversion. The flow diversion criterion was selected because it was readily identifiable everywhere within the simulation box and does a good job of representing the magnetopause surface. For our purposes, the magnetopause was defined as the maximum in the magnitude of the velocity gradient $(|\nabla V|)_{\max }$ lying downstream of the bow shock position. A twenty grid point (30 $\mathrm{R}_{\mathrm{J}}$ ) sphere around the planet was excluded when determining maxima. Figure 2 shows the contours of the magnitude of the velocity gradient from the Ogino-Walker simulation result, in the equatorial plane, at the nominal solar wind dynamic pressure of $0.090 \mathrm{nPa}$. Also shown on the plot are the model bow shock and magnetopause surfaces (discussed later) evaluated for this pressure.

[8] The scale sizes of the boundary surfaces and related parameters identified in the simulation results are summarized in Table 1 for both the magnetopause and bow shock. In the absence of an IMF, the magnetosphere shrinks as pressure increases. Polar flattening of the magnetosphere occurs at low pressure in the distended magnetosphere but as pressure increases, this effect diminishes and eventually reverses sign as the internal magnetospheric pressure is increasingly dominated by the quasi-dipolar Jovian magnetic field. The dawn side of the magnetosphere is inflated relative to the dusk side. The inflation, which occurs because the corotational flow opposes the solar wind flow at dawn while the flows are parallel at dusk, has been observed by Galileo [Kivelson et al., 2002] and is present in the MHD simulation [Ogino et al., 1998]. Dawn-dusk asymmetry of the magnetopause in the simulation becomes more pronounced as the pressure increases. In the absence of an IMF, the bow shock cross section in the dawn-dusk plane is nearly circular for all pressures. Analysis of gas dynamic flow characteristics [Stahara et al., 1989] indicates that polar flattening can affect shock shape only at large downstream distances where it must compete with other effects such as Mach cone shape as a function of IMF direction and strength. As the MHD simulation pressure increases, the shock stands closer to the magnetopause at the nose and further from the magnetopause in the dawndusk meridian plane.

[9] The system becomes much more complex for a nonvanishing IMF. Regardless of the IMF magnitude or orientation, both the magnetosphere and shock cavity are larger in all dimensions in the simulation for a given pressure when an IMF is present. With a northward (reconnecting) IMF, the shock stands closer to the magnetopause at the sub-solar point than in the case where no IMF is present; however, the opposite is true for a southward IMF. Table 1 shows that the size and shape of the magnetosphere are primarily controlled by changes in the solar wind dynamic pressure. The responses of both the bow shock and magnetopause to pressure changes are clearly systematic. For simplicity, this study only uses simulation results with vanishing IMF to characterize the boundary shapes. The magnetopause and bow shock are identified in the simulation results at different dynamic pressures in order to obtain shape models that change in response to dynamic pressure variations.

\section{Shape of the Bow Shock and Magnetopause}

[10] The collections of points identified as lying on either the bow shock or the magnetopause surface in the simulation were least squares fit to a second order polynomial of the form:

$$
z^{2}=A+B x+C x^{2}+D y+E y^{2}+F x y
$$

This form was selected because it allows both dawn-dusk asymmetry and polar flattening or steepening while imposing north-south symmetry. The Jupiter-centered coordinate system is defined such that $\mathrm{X}$ points towards the Sun, the $\mathrm{Z}$ axis is along the Jovian spin axis, positive in the direction of angular momentum, and Y completes the righthanded set (positive towards dusk). The form can reduce to any of the conic forms (ellipsoid, hyperboloid, paraboloid) if appropriate. In order to reduce numerical errors associated with the least squares fitting process all lengths were scaled by $120\left(\mathrm{R}_{\mathrm{J}} / 120\right)$. In order to avoid anomalies related to the simulation boundaries, the model boundary surfaces should 
BOW SHOCK

a

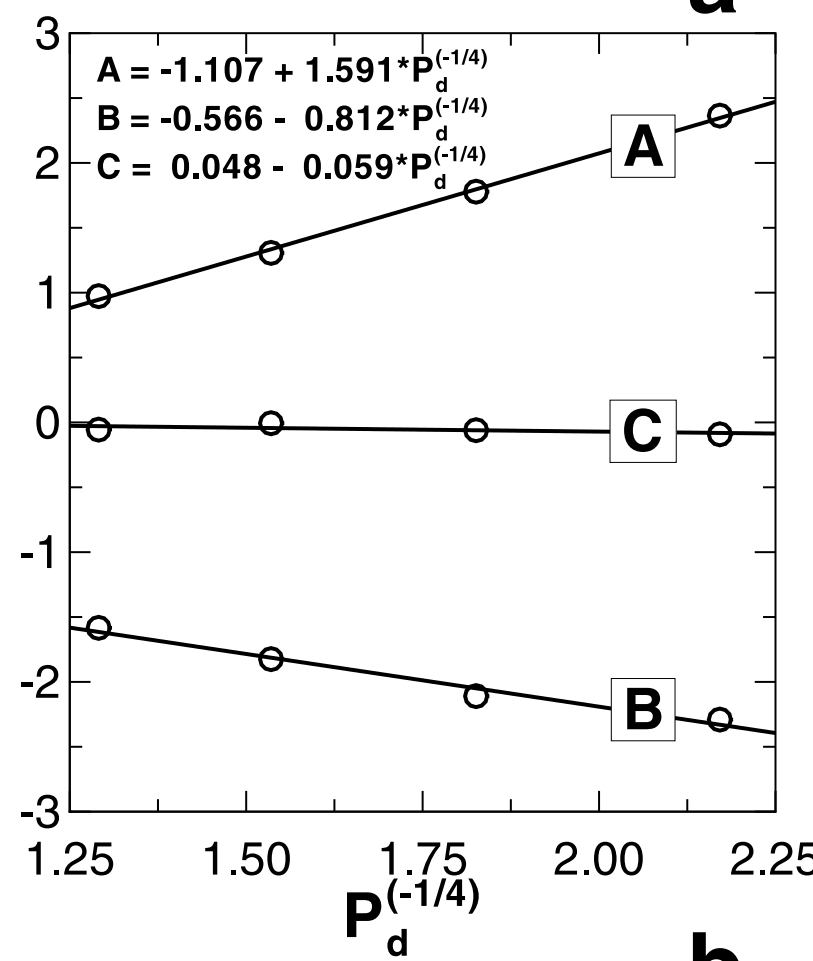

MAGNETOPAUSE
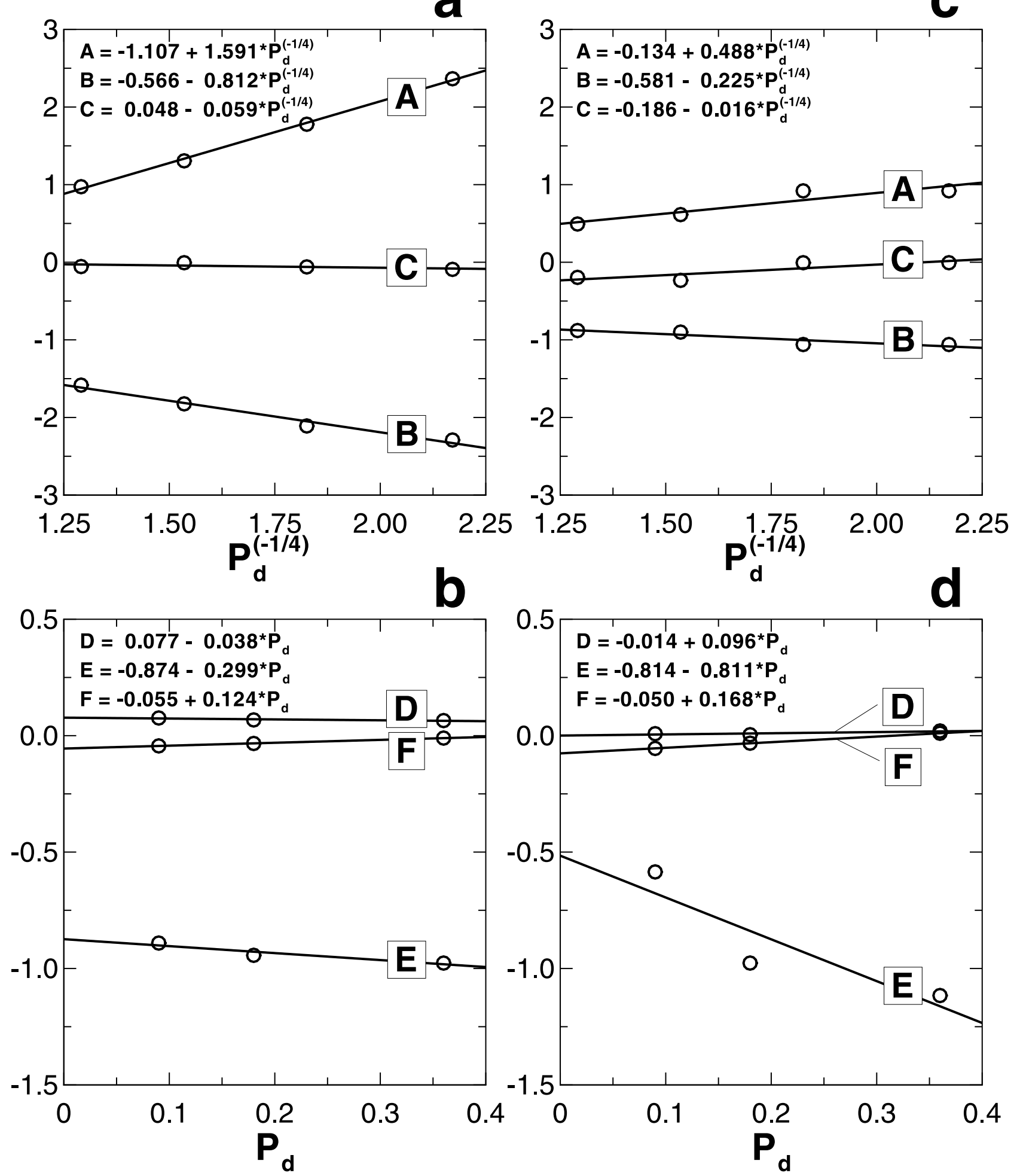

Figure 3. Pressure dependence of the model coefficients. Polynomial coefficients A-F (circles) of the boundary surface fits to the individual simulation results are plotted as linear functions (lines) of $P_{d}$ and $\mathrm{P}_{\mathrm{d}}{ }^{-1 / 4}$. Equations shown on panels a-d give the pressure dependence of the coefficients in equation (2) if distances are measured in units of $\mathrm{R}_{\mathrm{J}} / 120$. 

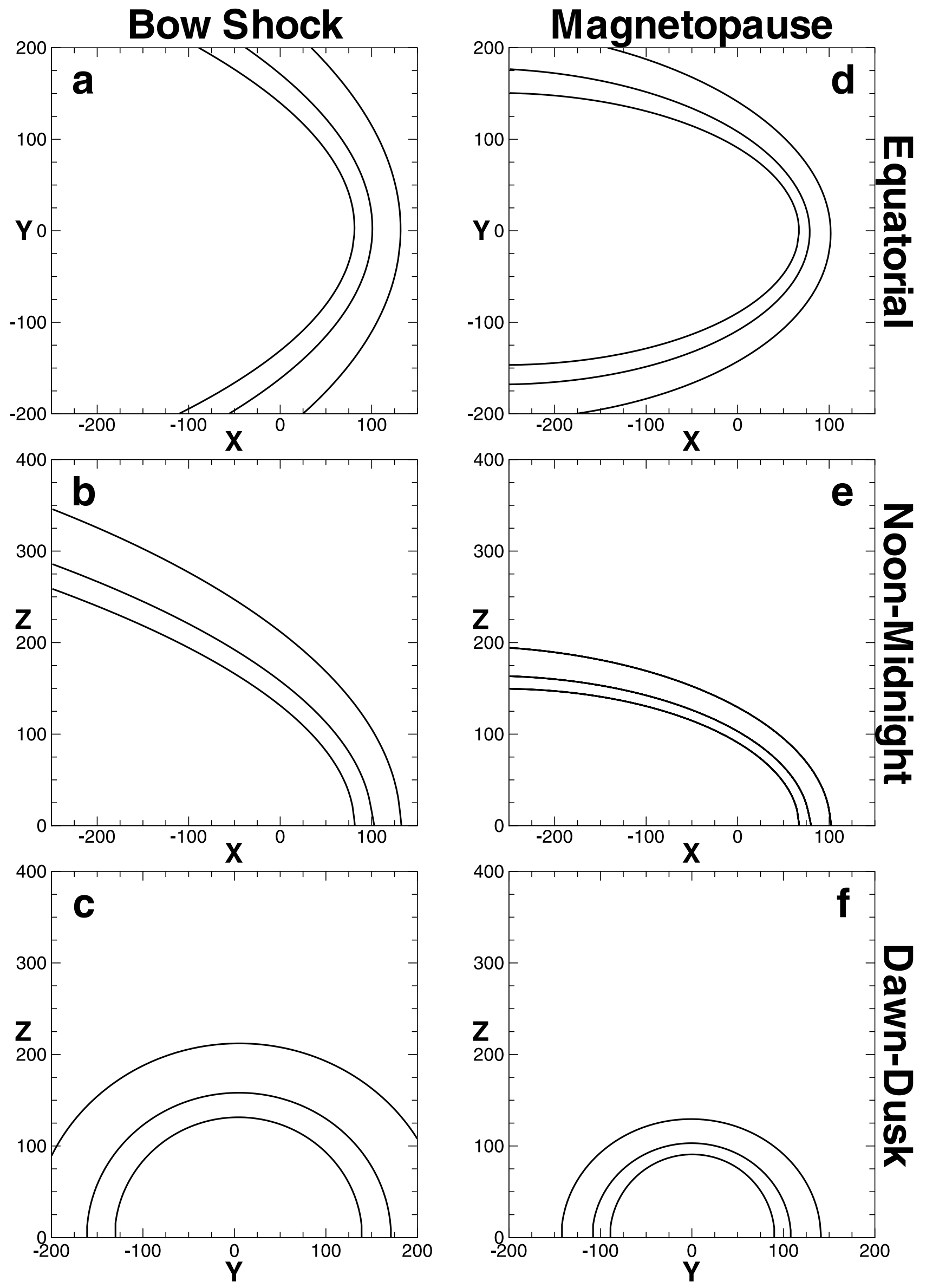
not be extended beyond $250 \mathrm{R}_{\mathrm{J}}$ in the anti-solar direction. The bow shock exits the simulation box for most pressures within $100 \mathrm{R}_{\mathrm{J}}$ downstream of the planet. Beyond this distance, the shock can be approximated by a Mach cone that is tangent to the model surface at about $\mathrm{X}=-100 \mathrm{R}_{\mathrm{J}}$ (see Slavin et al. [1984] or Petrinec and Russell [1997] for a discussion of the method and equations used for fitting asymptotic Mach cones in an obstacle tail region). The magnetopause remains within the simulation box beyond $250 \mathrm{R}_{\mathrm{J}}$ in the tailward direction, but it becomes more difficult to identify. In fitting the model surfaces, we weighted the simulation input more heavily at the nose than along the flanks in order to ensure a good fit in the region of greatest observational density at Jupiter. The selected functional form cannot reproduce both the blunt nose of the magnetopause and bow shock and their behavior at large distances in the tail. All of the boundary surfaces from the individual simulation least square fits close in the distant tail, and this also dictates that the fits be used only within $250 \mathrm{R}_{\mathrm{J}}$.

[11] After fitting the boundaries in the simulations, run at four values of dynamic pressure $\left(\mathrm{P}_{\mathrm{d}}\right)$, to the polynomial of equation (1), we fit the coefficients to power law models of $\mathrm{P}_{\mathrm{d}}$ and thereby obtain models of the magnetospheric boundaries as continuous functions of $\mathrm{P}_{\mathrm{d}}$.

$z^{2}=A\left(P_{d}\right)+B\left(P_{d}\right) x+C\left(P_{d}\right) x^{2}+D\left(P_{d}\right) y+E\left(P_{d}\right) y^{2}+F\left(P_{d}\right) x y$

The subsolar bow shock standoff distance has been shown to vary as a power law between $\mathrm{P}_{\mathrm{d}}^{-1 / 4}$ and $\mathrm{P}_{\mathrm{d}}{ }^{-1 / 5}$ [Slavin et al., 1985; Huddleston et al., 1998]. The magnetopause standoff distance varies slightly less with pressure than the bow shock standoff distance but is in the same range of power laws for the inferred solar wind dynamic pressure [Slavin et al., 1985]. The standoff distances in equation (1) are controlled by the coefficients A, B, and C. Figures $3 \mathrm{a}$ and $3 \mathrm{c}$ show that these coefficients depend linearly on $\mathrm{P}_{\mathrm{d}}{ }^{-1 / 4}$. The remaining polynomial coefficients, for both bow shock and magnetopause, are well described as linear functions of $\mathrm{P}_{\mathrm{d}}$ (Figures $3 \mathrm{~b}$ and $3 \mathrm{~d}$, respectively). At the lowest dynamic pressure $(0.045 \mathrm{nPa})$, the magnetopause impinges on the sides of the simulation box. Although boundary conditions correctly handle the situation where the bow shock passes through the edge of the box they are unsatisfactory for the magnetopause. For this reason, the lowest pressure case is used only to determine the pressure response of the coefficients that control the standoff distance, not the flaring angle. The equations for the pressure dependence of the coefficients in equation (2) are given in Figure 3.

[12] Figure 4 shows cross sections of the bow shock (left) and magnetopause (right) family of curves in a compressed, nominal, and expanded state. The boundaries are shown in the equatorial, noon-midnight, and dawn-dusk planes respectively. The cross-sections in Figure 4, as summarized in Table 1, are quite symmetric in the equatorial plane and nearly circular in the dawn-dusk plane. For the magnetopause, polar flattening is more pronounced at low pressure, and dawn-dusk asymmetry is greater at high pressure. Although asymmetry exists, it is not as pronounced in the simulations at steady state as might be expected. It is plausible that time varying solar wind conditions introduce additional asymmetries in the boundary shapes. Dynamic motions of the boundaries, including surface waves on the magnetopause, are observed in the simulation in time steps before equilibrium is reached, following changes in the solar wind or IMF input parameters to the simulation. However, the functional forms of the boundary surfaces presented here are representative only of the steady-state simulation results.

\section{Most Probable Bow Shock and Magnetopause Surfaces}

[13] In the previous section we developed a family of bow shock and magnetopause surfaces that were possible boundaries under steady state solar wind conditions. From the spacecraft observations we can establish the probability of finding the bow shock or magnetopause at different locations. Observed boundary crossings occur as a result of a combination of the transient response of the system to a change in the solar wind and the characteristics of the spacecraft trajectory and therefore boundary locations cannot be determined from the spatial distribution of a small number of crossings. However the fraction of the total observation time at any location in each magnetospatial region (magnetosphere, magnetosheath, or solar wind) provides additional information about the nominal boundary surface locations. For example, when the spacecraft is in the magnetosheath, the magnetosphere must be closer to the planet than the spacecraft and the bow shock must lie beyond the spacecraft. It is this information on which we base the models that are described in this paper.

[14] The trajectory of each spacecraft that crossed the Jovian system boundaries was sampled at ten minute resolution. Each sample was characterized as being in a magnetospatial region based on the published boundary crossings or direct analysis of the recent Galileo data (Pioneer [Intriligator and Wolfe, 1976]; Voyager [Lepping et al., 1981b]; Ulysses [Bame et al., 1992]; Galileo [Kivelson et al., 1997]). The ten-minute sampling rate was chosen in order to resolve short duration events with at least two samples. Only data acquired in the regions of model applicability that could be clearly assigned to a magnetospatial region were retained (see Figure 1). This excluded some of the observations from Voyager 2 outbound beyond $250 \mathrm{R}_{\mathrm{J}}$ in the anti-solar direction and some of the Galileo data from its capture orbit pass. Data were collected into "bins" by creating boundary surfaces defined by equation (2) with standoff distances that were separated by $4 R_{\mathrm{J}}$. All observations within the shell between two such surfaces were assigned to a bin identified by the average subsolar standoff distances of the two bounding surfaces. The fraction of the number of observa-

Figure 4. (opposite) Model bow shock and magnetopause shapes (columns) where the polynomial coefficients of equation (2) have been evaluated at the 10th (outer), 50th (middle), and 90th percentiles (inner) of observed solar wind dynamic pressure in three principal planes (rows). 
tions in each bin that was either inside or outside the magnetosphere was computed.

[15] As described above, ten-minute samples along the spacecraft trajectories were used in this study so that multiple closely spaced boundary crossings could be resolved in the data. In order to verify that our results are not corrupted by over sampling the trajectory data in time (samples not independent), the magnetospatial region data were sampled at different rates and then reanalyzed. Sample rates of 10 , 60 , and 600 minutes were tested. The observed distributions of magnetopause and bow shock locations are unaffected by changing the sampling rate. This does not prove that the tenminute samples are independent but it does show that the results are not compromised by over sampling.

[16] Sampling bias can also arise from the nature of the spacecraft trajectories. In some of the Galileo orbits, the spacecraft apogee may fall near a possible location of a boundary (MP or BS). Because the spacecraft is moving very slowly at these times, a large number of observations taken in a limited time interval may map to a small number of sub-solar distance bins. These observations may be dominated by a single set of solar wind conditions (IMF, dynamic pressure, etc.) instead of the full range of possible conditions. Since the objective is to determine the most probable boundary locations over all solar wind conditions, a large number of values from a single solar wind condition can bias the result. Similar bias is introduced by trajectories that are nearly parallel to the boundary models. Cassini observations (which have not been included in this analysis) would be subject to this type of bias since the trajectory closely parallels the bow shock family of curves after the spacecraft passes the dusk meridian. No attempt has been made to correct for the sampling biases associated with the spacecraft trajectories. The number of points in each bin is shown on the probability distribution plot (Figure 5) so that the regions affected by this type of bias can be identified.

[17] Figure 5 summarizes the characteristics of observations made within the region of model applicability for each standoff distance bin. The data points in Figure 5a show the fraction of observations that were in the solar wind at each nose standoff distance (based on the bow shock family of surfaces). Similarly, the points in Figure $5 \mathrm{~d}$ show the fraction of data in the magnetosphere versus the nose standoff distance (using the magnetopause surfaces). The confidence intervals for these data points were generated by taking ten independent random samples, each including ten percent of the total sample, to determine the range of values that might be obtained in each bin under different sampling conditions. The bounding error bars show the range of values obtained for each bin while the inner error bars mark the standard error of the mean obtained from the subsets of the sample. The solid lines show the fit to a Gaussian probability distribution and the dashed lines show the fit to a bimodal distribution function (sum of two Gaussians). The middle panels $(5 \mathrm{~b}, 5 \mathrm{e})$ show the number of samples in each bin.

[18] To show that the fraction of observations inside or outside a boundary measures the cumulative probability of the location of the boundary, we use the magnetopause data. We measure a conditional probability that the magnetopause is more distant than the current bin, given that the observing spacecraft lies within the bin $P(A \mid B)$. If $P(A)$ is the proba- bility that the magnetopause lies beyond the bin and $P(B)$ is the probability that the spacecraft is within the bin, then Bayes' theorem allows us to convert the conditional probability into a joint probability $P(A \& B)$.

$$
P(A \mid B)=P(A \& B) / P(B)
$$

However, since the location of the magnetopause is independent of the position of the observing spacecraft, the joint probability is just the product of the individual event probabilities. Thus:

$$
P(A \mid B)=P(A \& \mathrm{~B}) / P(B)=P(A) \cdot P(B) / P(B)=P(A)
$$

[19] The probability density distribution of the location of magnetopause or bow shock is the derivative of the cumulative probability. Figures $5 \mathrm{c}$ and $5 \mathrm{f}$ show the probability densities for the bow shock and magnetopause plotted along the Jupiter-Sun line. The derivatives (points) shown in Figure 5 were computed numerically from the smoothed data bins shown in panels $5 \mathrm{a}$ and $5 \mathrm{~d}$. The probability density for the magnetopause location has two well-defined peaks, i.e. a bimodal distribution. The observed bow shock probability density also appears bimodal (or polymodal) but a single distribution function cannot be ruled out within the uncertainties of the fit. In panel $5 \mathrm{c}$ and $5 \mathrm{f}$, the dashed line shows the fit of a bimodal distribution (sum of two Gaussian distributions) to the data while the solid line shows a Gaussian distribution fit. The moments of the fit distributions are also shown in Figures 5a and 5d. Subscripts are used to distinguish the components of the bimodal distribution. Values without subscripts are the moments of the single distribution function fit. For the magnetopause, both distributions are weighted evenly. For the bow shock, the "compressed state" distribution is weighted more heavily $(70 \%)$ than the "expanded state". The distribution weights were free parameters of the bimodal fit. The weighting factor determines the transition point from one probability distribution to the other.

[20] The quality of both the Gaussian distribution and bimodal distribution fits were investigated in a variety of ways. The Kolmogorov-Smirnov (K-S) test compares the maximum difference in an observed cumulative probability of a variable with that of the fitted probability distribution function as a measure of the "goodness" of the fit. For the magnetopause model, the K-S test indicates that the maximum deviation between the observed bin means and the fitted bimodal distribution function is expected more than $99.9 \%$ of the time. However, when compared to the Gaussian distribution function, deviations of the observed size would occur through sampling errors only $37.8 \%$ of the time. For the bow shock, the K-S test result shows that the observed deviations would be expected $99.9 \%$ of the time for either the bimodal or Gaussian distribution functions. The F-test can be used to determine the statistical significance of additional parameters (degrees of freedom) in a fitting function. The F-test was used to compare the variances in the deviations from the Gaussian distribution to those of the bimodal distribution. The results show that the improvement in the fit to the magnetopause obtained by using the bimodal distribution is significant at greater than the $99.9 \%$ confidence level. 


\section{Bow Shock}
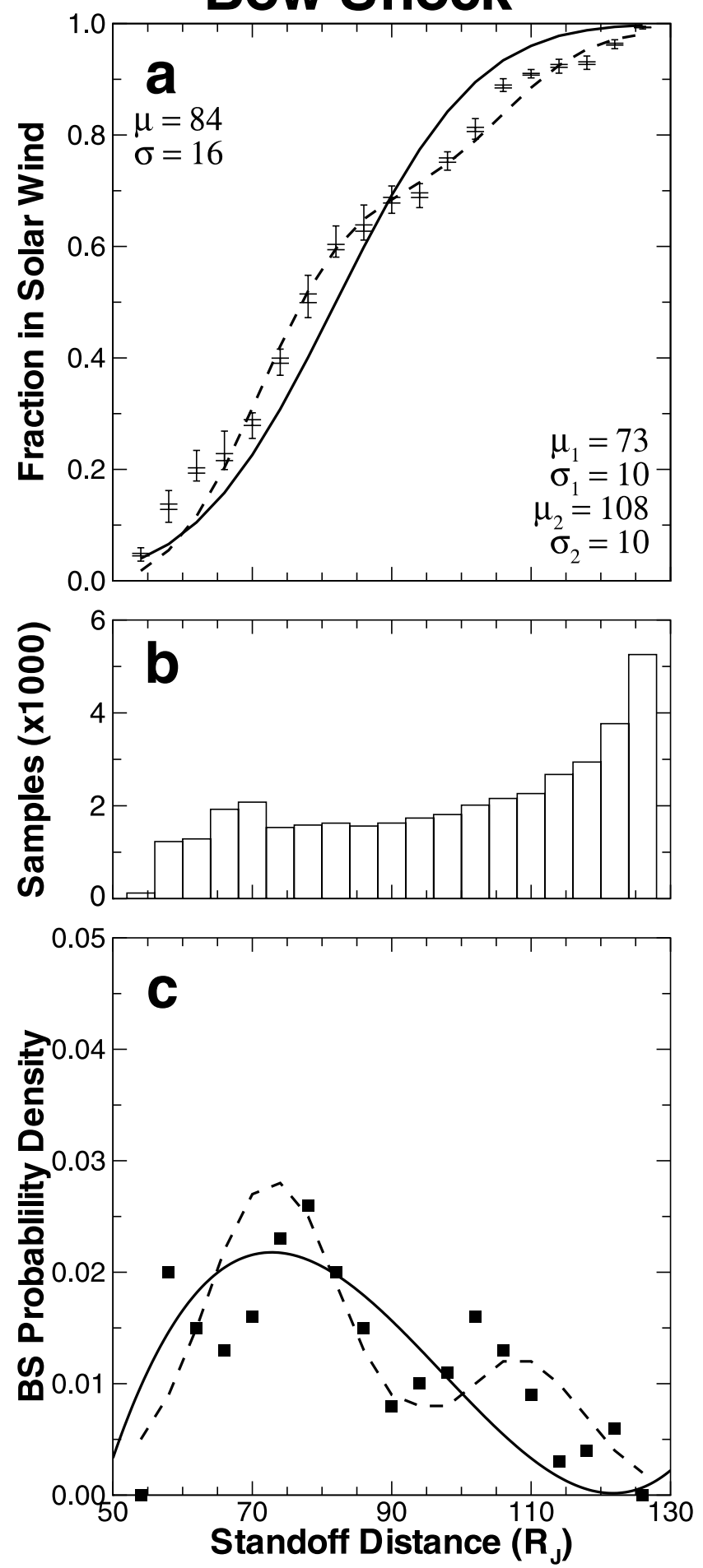

Magnetopause
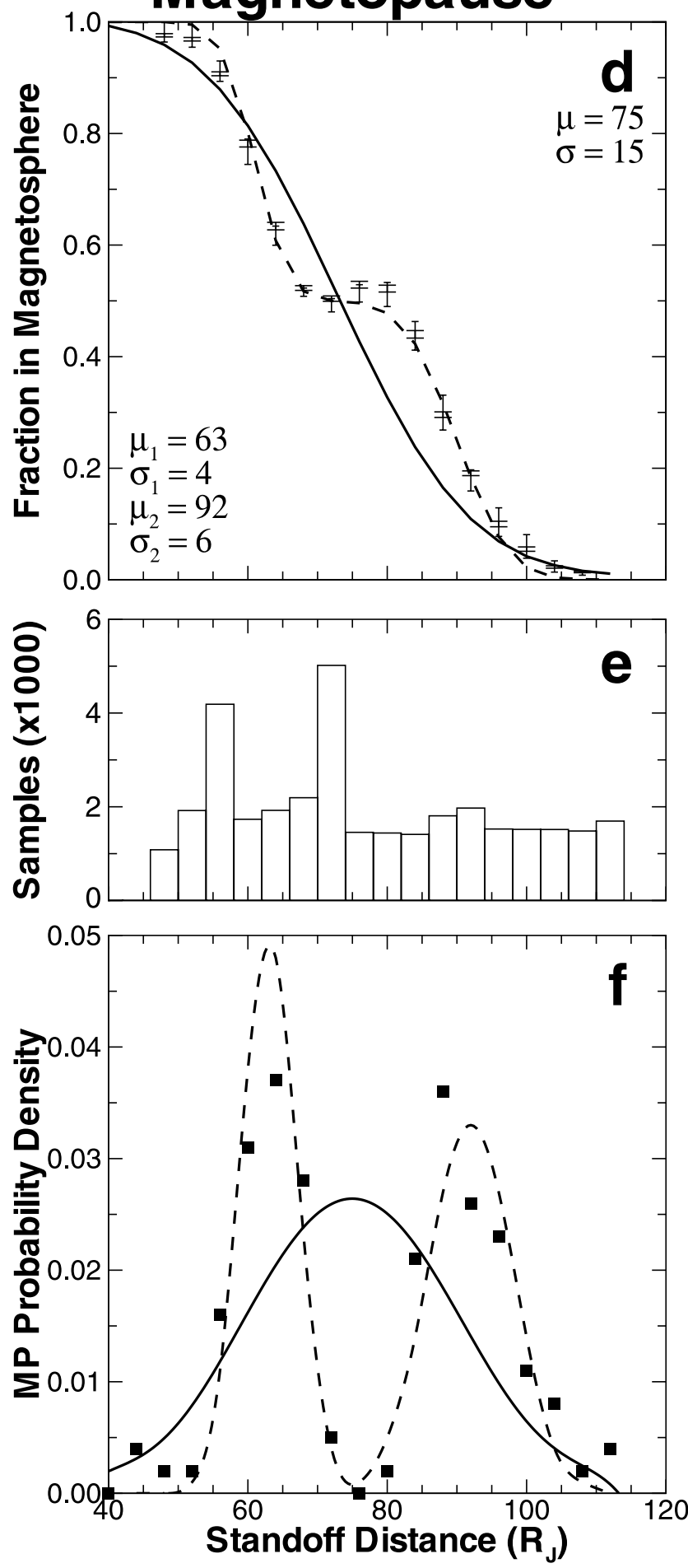

Figure 5. Top panels show the fraction of the observations outside the bow shock (a) or inside the magnetopause (d) versus standoff distance. Distribution function moments and fits are also shown. Middle panels show the number of observations in each standoff distance bin. Bottom panels show the probability densities (solid boxes) derived by numerical differentiation of the data in the top panels. See text for discussion.

However, the F-test indicates that the additional free parameters of a bimodal distribution for the bow shock data are not statistically significant. In this case, the improvement to the fit obtained by using the bimodal distribution is significant at only the $89.8 \%$ confidence interval.

[21] Lastly, in order to verify that the bimodal signature in the magnetopause does not arise because of a sampling bias 
Table 2. Solar Wind Dynamic Pressures Required in the Polynomial Fits to Reproduce Bow Shock and Magnetopause Surfaces at the Observed Median, Quartile, and Decile Probability Levels

\begin{tabular}{lcc}
\hline & Magnetopause, $\mathrm{nPa}$ & Bow Shock, nPa \\
\hline 10th percentile & 0.030 & 0.063 \\
25th percentile & 0.048 & 0.111 \\
50th percentile & $0.209^{\mathrm{a}}$ & 0.258 \\
75th percentile & 0.383 & 0.382 \\
90th percentile & 0.518 & 0.579 \\
\hline
\end{tabular}

${ }^{a}$ Defines a low-probability magnetopause surface.

related to the bin size, the data were analyzed by using different bin sizes from $2-20 \mathrm{R}_{\mathrm{J}}$. The bimodal signature persists for all bin sizes up to $15 \mathrm{R}_{\mathrm{J}}$ or half of the separation of the means of the two distributions that form the bimodal signature. In other words, the bimodal distribution signature persists in the data until the scale size of the bin approaches the scale size of the system.

[22] The polynomial coefficients of the bow shock and magnetopause shape models vary with solar wind dynamic pressure. Table 2 provides the pressure values of the surfaces with standoff distances corresponding to different probabilities of being inside the magnetopause or inside the bow shock, respectively. These probability surfaces are defined by equation (2) with the coefficients corresponding to pressure values from Table 2. Because the probability varies monotonically with distance from Jupiter, the bow shock location is usefully described in terms of the percentage of time an observer would be inside the bow shock at a given distance. On the other hand, the bimodal distribution of the magnetopause location makes the probability of observing the magnetopause near the $50^{\text {th }}$ percentile surface low. If the subscript $(i)$ is used to distinguish the compressed and expanded subsolar distances in the bimodal distribution, then the magnetopause location is more accurately described by using one standard deviation $\left(\sigma_{i}\right)$ bands about the means $\left(\mu_{i}\right)$. The range of dynamic pressures that correspond to the compressed magnetopause location are 0.306 $(+0.108,-0.078) \mathrm{nPa}$. For the expanded state, the pressures are $0.039(+0.020,-0.014) \mathrm{nPa}$. Similarly, the bow shock locations of the somewhat speculative bimodal distribution are generated from equation (2) by using pressures of 0.315 $(+0.148,-0.104) \mathrm{nPa}$ for the compressed and 0.070 $(+0.041,-0.028) \mathrm{nPa}$ for the expanded states.

[23] Figure 6 compares the models developed here with the Huddleston [Huddleston et al., 1998] and Voyager [Lepping et al., 1981a] magnetopause and bow shock models. In all panels, the Huddleston models (high, low solar wind dynamic pressure) are represented with solid lines, the Voyager models with dashed lines, the newly developed models are shaded to show a range of probabilities in the boundary location. Open circles indicate actual boundary crossings observed. In places where there are multiple closely spaced crossings, the open circles appear to be filled. There is a substantial spread in the dawn-dusk dimension of the observed dayside crossings suggesting that the upstream boundary is rather blunt. The Huddleston models of the bow shock and magnetopause for high solar wind dynamic pressure conditions are both slightly more compressed at the nose but are otherwise

\section{Bow Shock}

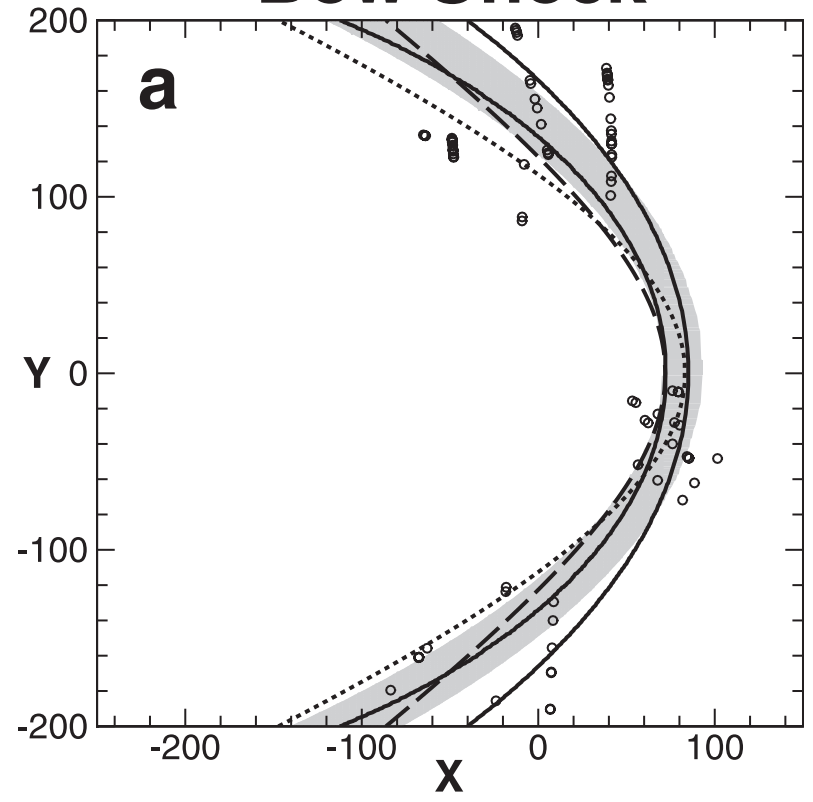

Magnetopause

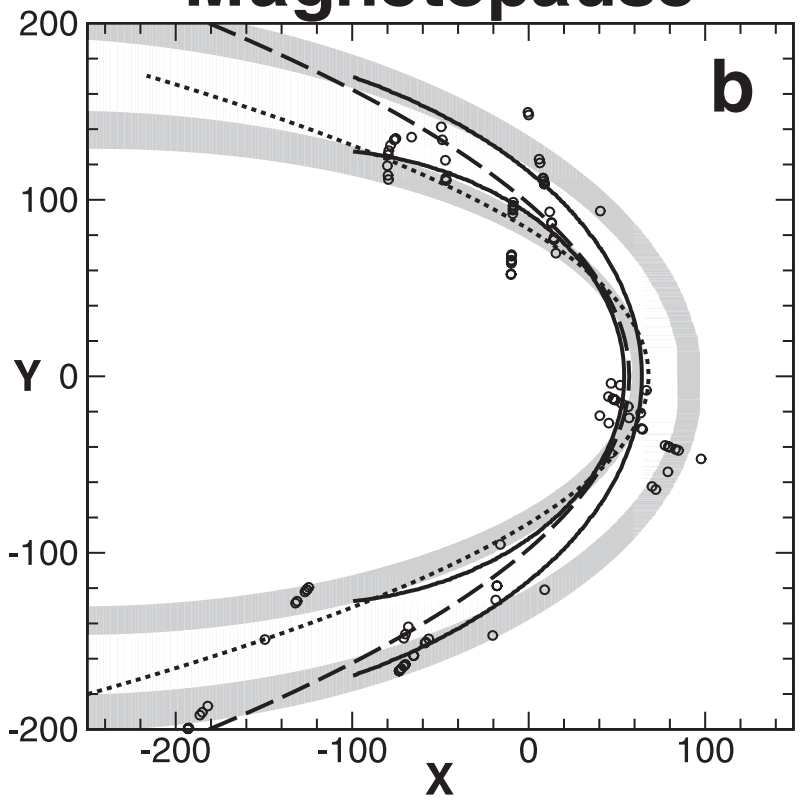

Figure 6. Comparison of the probabilistic bow shock (a) and magnetopause (b) models with previous models. The area between the 25 th and 75 th percentiles of the probability of being in the solar wind is shaded in panel (a). In panel (b), shading indicates one sigma bands about the means of the bimodal distribution in the probability of being inside the magnetosphere. Solid lines show the high and low pressure models of Huddleston et al. [1998]. Dashed lines show the Voyager models [Lepping et al., $1981 \mathrm{a}]$. 
quite similar to the shape models presented here. However, for the magnetopause at low pressure, the Huddleston models are significantly compressed on the dayside relative to our models under similar conditions. Magnetopause crossings are observed well upstream of the low pressure Huddleston model. The Voyager models are less blunt on the dayside and are more flared throughout, than either our models or the Huddleston models. The Voyager magnetopause models do not fit the distant dayside crossing observations. In addition, the Voyager models suggest that as the standoff distances are increased the flaring angles decrease, causing the models to cross. Both the Voyager bow shock and magnetopause models have this characteristic, which is not observed in the Ogino-Walker MHD simulation results.

\section{Solar Wind Near Jupiter}

[24] In order to understand if the probability density distributions of the Jovian magnetopause and bow shock relate to the statistical properties of the solar wind, we have re-examined the solar wind characteristics in the vicinity of Jupiter. We used one hour averaged data from the Coordinated Heliospheric Observations (COHO) database at the National Space Science Data Center (NSSDC, COHOWeb) from both Pioneers, both Voyagers, and Ulysses when the spacecraft were within 0.25 AU of Jupiter. Galileo does not acquire plasma data in the solar wind due to the downlink limitations imposed by the spacecraft high gain antenna failure. Data analysis intervals were selected to span integral numbers of solar rotations. Selected solar rotations were required to have at least $70 \%$ data coverage for each of the key parameters. Ulysses data were constrained to lie near the ecliptic plane by selecting data primarily from the inbound to Jupiter portion of the flyby. Four solar rotations of data were analyzed from the two Pioneers and Ulysses; only three rotations of data met the selection criteria for the two Voyagers.

[25] Solar cycle variations and statistical properties of the solar wind have been extensively studied [Gazis, 1996; Slavin et al., 1986; Winterhalter et al., 1990; Richardson et al., 1996; Riley et al., 1997]. However, most of these studies have focused on topics such as the properties near Earth, radial gradients, or variations with heliographic latitude. The solar wind in the vicinity of Jupiter has long been known to vary from relatively quiet, slow solar wind to compressed, turbulent regions such as corotating interaction regions (CIRs) and coronal mass ejections (CMEs) [Smith and Wolfe, 1979]. The relative fraction of disturbed solar wind attributable to CMEs increases at solar maximum and decreases at solar minimum [Webb and Howard, 1994]. Slavin et al. [1985] examined the solar wind properties (Alfvén Mach number, sonic Mach number, dynamic pressure) as observed by Pioneer 10 within 0.1 AU of Jupiter, excluding data inside the bow shock. They found that the dynamic pressure and sonic Mach numbers matched values expected from extrapolating near Earth results to Jupiter's distance from the Sun but that the Alfvénic Mach number was higher than predicted. The authors reasoned that there could be deficiencies in the scaling laws but these effects could also arise either from solar cycle variability or inadequate temporal coverage. However, Slavin et al. [1985] did not distinguish between quiet and disturbed solar wind in their analysis. We have classified data as belonging to either undisturbed (normal, quiet) or disturbed (CIR, CME) solar wind conditions and computed statistics for each solar wind component individually in addition to computing them for the entire data intervals.

[26] Figure 7 shows selected parameters from the Pioneer 10 spacecraft for three solar rotations after the Jupiter flyby. Shading is used to identify disturbed regions in the figure. Within these regions, the IMF is compressed and turbulent, the dynamic pressure is high, the solar wind velocity is increasing, and the Alfvén Mach number is low. Conversely, in the intervening intervals, the IMF is weak and steady, the dynamic pressure is generally low, the velocity is decreasing, and the Alfvén Mach number is high and irregular. Disturbed regions are identified primarily on the basis of bounding forward and reverse shocks that are readily identifiable in the IMF data. In some instances, there is more than one shock that could be used to identify the boundaries. In these instances, the solar wind velocity is used to select between possible boundary events.

[27] Table 3 summarizes the statistical properties of the solar wind within disturbed regions, in quiet regions, and for the complete time intervals (all) for five spacecraft. The solar wind parameters tabulated include the IMF strength (B), the dynamic pressure $\left(\mathrm{P}_{\mathrm{d}}\right)$, the Alfvén Mach number $\left(\mathrm{M}_{\mathrm{a}}\right)$, and the plasma beta $(\beta)$. When computing $\mathrm{M}_{\mathrm{a}}, \mathrm{P}_{\mathrm{d}}$ and $\beta$, we have assumed that the solar wind contains $4 \% \mathrm{He}^{++}$ with a temperature of 3.5 times the proton temperature. This approximation allows our results to be compared directly to those previously reported by Slavin et al. [1985].

$$
\begin{aligned}
P_{d} & =1.16 n m_{p} V^{2} \\
M_{a} & =V\left(4 \pi 1.16 n m_{p}\right)^{1 / 2} / B \\
\beta & =2 \mu_{o} n k\left(1.14 T_{p}+1.08 T_{e}\right) / B^{2}
\end{aligned}
$$

The statistical parameters we report are the mean, median, mode, standard deviation and number of samples (n) in each data set. There are many missing data points in the $\mathrm{COHO}$ database so the number of samples is given for each individual parameter. The number reported next to the words "disturbed", "quiet", and "all" are the total number of possible data points in the identified time intervals. In addition, data are organized by solar cycle. Both Pioneer spacecraft were in the vicinity of Jupiter near solar minimum so their results have been combined to provide better statistics near solar minimum. The two Voyager spacecraft reached Jupiter near solar maximum so those data are also presented both individually and combined into a set of conditions applicable to solar maximum. Ulysses was at Jupiter two years after the 1989 solar maximum. These data are not included in either the solar minimum or maximum data sets but are included in the average solar wind properties.

[28] There are many interesting results in Table 3. The parameter distributions observed by the two Pioneer spacecraft are remarkably similar although one year separated the two sets of observations. The Voyager passes are also very similar but they were nearly contemporaneous. Disturbed and quiet solar winds clearly have distinct and separate statistical distributions for all parameters $\left(B, P_{d}, M_{a}\right.$, and $\left.\beta\right)$, spacecraft, and times within the solar cycle. The IMF 


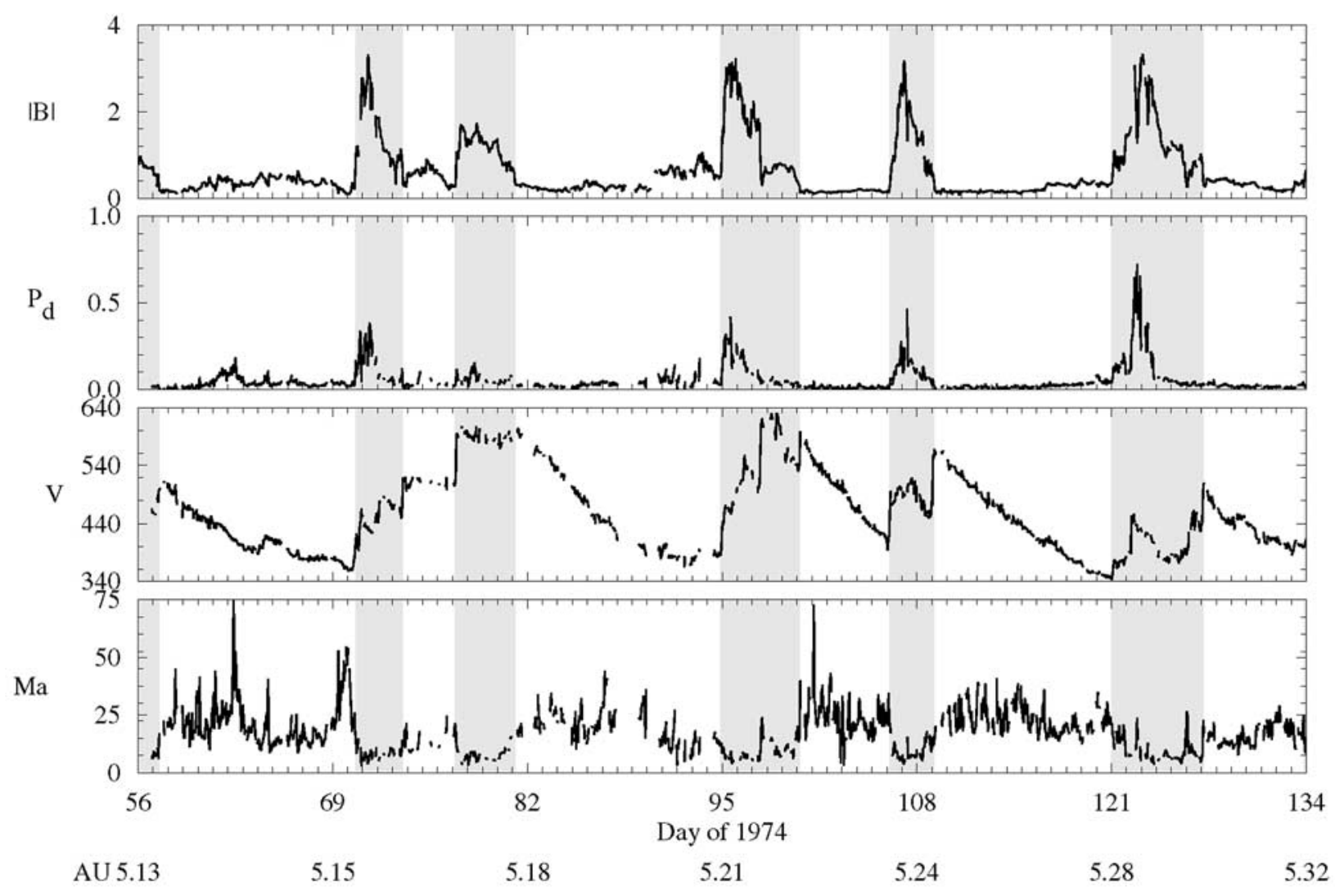

Figure 7. Magnetic field strength $(\mathrm{B}, \mathrm{nT})$, solar wind dynamic pressure $\left(\mathrm{P}_{\mathrm{d}}, \mathrm{nPa}\right)$, velocity $(\mathrm{V}, \mathrm{km} / \mathrm{s})$, and Alfvén Mach number $\left(\mathrm{M}_{\mathrm{A}}\right)$ from Pioneer 10 are plotted versus time for three solar rotations after the Jupiter encounter. Disturbed regions identified by using the magnetic field data are shaded. Heliocentric distances are shown using secondary labels for major tick marks on the time axis.

strength varies by a factor of 4-5 between quiet and disturbed regions but varies by about only $25 \%$ over the solar cycle. The latter result is in agreement with near Earth observations [Slavin et al., 1986; Winterhalter et al., 1990]. The difference between the mean dynamic pressures in the disturbed $(0.20 \mathrm{nPa})$ versus the quiet $(0.04 \mathrm{nPa})$ solar winds at solar minimum is more than twice the difference $(0.13$, $0.05 \mathrm{nPa}$ ) observed at solar maximum. Slavin [Slavin et al., 1985] noted that $\mathrm{M}_{\mathrm{a}}$ observed by Pioneer 10 was larger than expected based on scaling values measured near Earth to 5 A.U. The data in Table 3 suggest that this inconsistency could be the result of Slavin's selection of data near solar minimum. Both Pioneer 10 and 11 found similar values of $\mathrm{M}_{\mathrm{a}}$ at solar minimum (20.1) but the average over all spacecraft (15.7) is consistent with scaling law expectations. Plasma beta at solar minimum is nearly double the values observed during solar maximum whereas disturbed and quiet regions are within $20 \%$ of each other. With the exception of plasma beta, the parameters studied differ more between the quiet and disturbed solar wind (factors of $2-5)$ than between solar minimum and maximum (20$50 \%$ ). However, the fraction of the solar rotation that is categorized as disturbed increases from 33\% at solar minimum to $45 \%$ at solar maximum. This difference may be the most significant solar cycle variation in terms of its poten- tial impact on the Jovian system boundary locations. We have analyzed the characteristics of the solar wind dynamic pressure near Jupiter in more detail than other parameters because it is relevant to the models of the magnetospheric boundaries. We find that when data from all spacecraft are considered, the solar wind dynamic pressure is well represented by a sum of two lognormal distributions. The lognormal probability density function, i.e. the natural logarithm of $x=P_{d}[n P a]$ is normally distributed, is given by

$$
P(x)=\frac{1}{\sigma x \sqrt{2 \pi}} \exp \left(\frac{-\left(\log _{e}(x)-\mu\right)^{2}}{2 \sigma^{2}}\right) .
$$

Variables that naturally span several orders of magnitude are commonly found to have lognormal distributions. The observed distribution, although generated by summing two individual distributions with moments $\mu_{1}=-1.915, \sigma_{1}=$ 0.780 and $\mu_{2}=-3.237, \sigma_{2}=0.615$, is not truly bimodal since only one clearly defined peak is present in the distribution. The $\mathrm{K}-\mathrm{S}$ test indicates that the fit of the distribution sum is significant at greater than the $95 \%$ confidence interval whereas the single lognormal distribution fit is significant only at the $66 \%$ confidence level. A 
Table 3. Summary of Solar Wind Parameter Statistics Derived From the COHO Database Over Integral Numbers of Solar Rotations ${ }^{\mathrm{a}}$

\begin{tabular}{|c|c|c|c|c|c|c|c|c|c|c|c|c|c|c|c|}
\hline & \multicolumn{5}{|c|}{ Pioneer 10 Disturbed (741) } & \multicolumn{5}{|c|}{ Pioneer 11 Disturbed (898) } & \multicolumn{5}{|c|}{ All Solar Minimum Disturbed (1639) } \\
\hline & Mean & Med & StDev & Mode & $\mathrm{n}$ & Mean & Med & StDev & Mode & $\mathrm{n}$ & Mean & Med & StDev & Mode & $\mathrm{n}$ \\
\hline $\mathrm{B}, \mathrm{nT}$ & 1.3 & 1.16 & 0.71 & 0.76 & 712 & 1.59 & 1.36 & 0.97 & 1.46 & 815 & 1.45 & 1.25 & 0.87 & 0.76 & 1527 \\
\hline $\mathrm{Pd}, \mathrm{nPa}$ & 0.17 & 0.12 & 0.15 & 0.09 & 457 & 0.22 & 0.16 & 0.22 & 0.15 & 711 & 0.2 & 0.15 & 0.2 & 0.08 & 1168 \\
\hline $\mathrm{Ma}$ & 10.6 & 9.5 & 5 & 8 & 444 & 11.4 & 10.9 & 5 & 10 & 674 & 11.1 & 10.3 & 5 & 8 & 1118 \\
\hline \multirow[t]{3}{*}{$\underline{B e t a}$} & 0.68 & 0.28 & 1.7 & 0.07 & 444 & 0.38 & 0.28 & 0.41 & 0.1 & 674 & 0.5 & 0.28 & 1.13 & 0.1 & 1118 \\
\hline & \multicolumn{5}{|c|}{ Quiet (1755) } & \multicolumn{5}{|c|}{ Quiet (1598) } & \multicolumn{5}{|c|}{ Quiet (3353) } \\
\hline & Mean & Med & StDev & Mode & $\mathrm{n}$ & Mean & Med & StDev & Mode & $\mathrm{n}$ & Mean & Med & StDev & Mode & $\mathrm{n}$ \\
\hline $\mathrm{B}, \mathrm{nT}$ & 0.34 & 0.3 & 0.18 & 0.16 & 1655 & 0.32 & 0.26 & 0.17 & 0.2 & 1459 & 0.33 & 0.28 & 0.17 & 0.18 & 3114 \\
\hline $\mathrm{Pd}, \mathrm{nPa}$ & 0.04 & 0.03 & 0.03 & 0.02 & 1320 & 0.05 & 0.03 & 0.04 & 0.03 & 1303 & 0.04 & 0.03 & 0.04 & 0.02 & 2623 \\
\hline & 23.6 & 22.3 & 9.1 & 16 & 1302 & 24.4 & 23.8 & 8 & 24 & 1272 & 24 & 23.1 & 8.6 & 20 & 2574 \\
\hline \multirow[t]{3}{*}{ Beta } & 0.59 & 0.38 & 0.76 & 0.16 & 1302 & 0.58 & 0.38 & 0.96 & 0.01 & 1272 & 0.59 & 0.38 & 0.87 & 0.12 & 2574 \\
\hline & \multicolumn{5}{|c|}{ All (2496) } & \multicolumn{5}{|c|}{ All (2496) } & \multicolumn{5}{|c|}{ All (4992) } \\
\hline & Mean & Med & StDev & Mode & $\mathrm{n}$ & Mean & Med & StDev & Mode & $\mathrm{n}$ & Mean & Med & StDev & Mode & $\mathrm{n}$ \\
\hline $\mathrm{B}, \mathrm{nT}$ & 0.63 & 0.4 & 0.61 & 0.16 & 2366 & 0.77 & 0.4 & 0.85 & 0.2 & 2274 & 0.7 & 0.4 & 0.74 & 0.18 & 4640 \\
\hline $\mathrm{Pd}, \mathrm{nPa}$ & 0.07 & 0.04 & 0.1 & 0.02 & 1776 & 0.11 & 0.05 & 0.16 & 0.03 & 2014 & 0.09 & 0.05 & 0.13 & 0.02 & 3790 \\
\hline $\mathrm{Ma}$ & 20.3 & 19.3 & 10 & 16 & 1745 & 19.9 & 19.4 & 9.4 & 10 & 1946 & 20.1 & 19.4 & 9.7 & 20 & 3691 \\
\hline \multirow[t]{3}{*}{ Beta } & 0.62 & 0.35 & 1.08 & 0.07 & 1745 & 0.51 & 0.33 & 0.82 & 0.01 & 1946 & 0.56 & 0.34 & 0.95 & 0.07 & 3691 \\
\hline & \multicolumn{5}{|c|}{ Voyager 1} & \multicolumn{5}{|c|}{ Voyager 2} & & All & olar Max & mum & \\
\hline & & & turbed $(8$ & & & & & turbed ( & & & & & urbed (1 & 88) & \\
\hline Mean & Med & StDev & Mode & $\mathrm{n}$ & Mean & Med & StDev & Mode & $\mathrm{n}$ & Mean & Med & StDev & Mode & $\mathrm{n}$ & \\
\hline $\mathrm{B}, \mathrm{nT}$ & 1.28 & 1.23 & 0.39 & 0.98 & 811 & 1.51 & 1.34 & 0.72 & 0.86 & 734 & 1.39 & 1.27 & 0.58 & 0.98 & 1545 \\
\hline $\mathrm{Pd}, \mathrm{nPa}$ & 0.1 & 0.08 & 0.08 & 0.04 & 771 & 0.16 & 0.11 & 0.13 & 0.07 & 716 & 0.13 & 0.09 & 0.11 & 0.04 & 1487 \\
\hline $\mathrm{Ma}$ & 8.3 & 7.7 & 4.1 & 6 & 765 & 10.1 & 8.9 & 5.5 & 8 & 716 & 9.2 & 8.2 & 4.9 & 6 & 1481 \\
\hline Beta & 0.2 & 0.09 & 0.54 & 0.02 & 765 & 0.35 & 0.2 & 0.69 & 0.16 & 716 & 0.27 & 0.14 & 0.62 & 0.09 & 1481 \\
\hline & & & uiet $(9$ & & & & & uiet $(108$ & & & & & uiet $(205$ & & \\
\hline & Mean & Med & StDev & Mode & $\mathrm{n}$ & Mean & Med & StDev & Mode & $\mathrm{n}$ & Mean & Med & StDev & Mode & $\mathrm{n}$ \\
\hline $\mathrm{B}, \mathrm{nT}$ & 0.51 & 0.45 & 0.27 & 0.4 & 955 & 0.5 & 0.5 & 0.24 & 0.3 & 1077 & 0.51 & 0.47 & 0.26 & 0.3 & 2032 \\
\hline $\mathrm{Pd}, \mathrm{nPa}$ & 6 & 0.04 & 0.07 & 0.04 & 937 & 0.04 & 0.03 & 0.03 & 0.02 & 1067 & 0.05 & 0.04 & 0.05 & 0.02 & 2004 \\
\hline Ma & 18.4 & 16.6 & 10.2 & 16 & 933 & 16.3 & 14.1 & 8.4 & 10 & 1067 & 17.3 & 15.4 & 9.4 & 12 & 2000 \\
\hline Beta & 0.38 & 0.21 & 1.02 & 0.11 & 933 & 0.26 & 0.15 & 0.58 & 0.09 & 1067 & 0.32 & 0.17 & 0.82 & 0.09 & 2000 \\
\hline & & & All (1872 & & & & & All (1872 & & & & & All (3744 & & \\
\hline & Mean & Med & StDev & Mode & $\mathrm{n}$ & Mean & Med & StDev & Mode & $\mathrm{n}$ & Mean & Med & StDev & Mode & $\mathrm{n}$ \\
\hline $\mathrm{B}, \mathrm{nT}$ & 0.86 & 0.76 & 0.51 & 0.4 & 1766 & 0.91 & 0.69 & 0.7 & 0.3 & 1811 & 0.89 & 0.72 & 0.61 & 0.3 & 3577 \\
\hline $\mathrm{Pd}, \mathrm{nPa}$ & & 0.05 & 0.07 & 0.04 & 1708 & 0.09 & 0.0 & 0.1 & 0.03 & 1783 & 0.08 & 0.05 & 0.09 & 0.03 & 3491 \\
\hline $\mathrm{Ma}$ & 13.9 & 11.7 & 9.5 & 6 & 1698 & 13.8 & 11.8 & 8 & 8 & 1783 & 13.8 & 11.7 & 8.8 & 8 & 3481 \\
\hline Beta & 0.3 & 0.14 & 0.84 & 0.02 & 1698 & 0.3 & 0.16 & 0.63 & 0.07 & 1783 & 0.3 & 0.15 & 0.74 & 0.09 & 3481 \\
\hline & & Ulysses & Disturbe & (1091) & & & & & & & & 11 Space & aft Distu & bed (441 & \\
\hline & Mean & Med & StDev & Mode & $\mathrm{n}$ & & & & & & Mean & Med & StDev & Mode & $\mathrm{n}$ \\
\hline $\mathrm{B}, \mathrm{nT}$ & 1.78 & 1.59 & 0.79 & 1.48 & 1088 & & & & & & 1.53 & 1.39 & 0.78 & 1.12 & 4160 \\
\hline $\mathrm{Pd}, \mathrm{nPa}$ & 0.19 & 0.14 & 0.15 & 0.08 & 1072 & & & & & & 0.17 & 0.13 & 0.15 & 0.07 & 3671 \\
\hline $\mathrm{Ma}$ & 9.1 & 8.5 & 4.2 & 8 & 1072 & & & & & & 9.7 & 8.8 & 4.8 & 6 & 3687 \\
\hline Beta & 0.47 & 0.32 & 0.57 & 0.13 & 1072 & & & & & & 0.41 & 0.22 & 0.79 & 0.09 & 3671 \\
\hline & & & uiet (140 & & & & & & & & & & uiet (681 & & \\
\hline & Mean & Med & StDev & Mode & $\mathrm{n}$ & & & & & & Mean & Med & StDev & Mode & $\mathrm{n}$ \\
\hline $\mathrm{B}, \mathrm{nT}$ & 0.62 & 0.56 & 0.26 & 0.4 & 1400 & & & & & & 0.44 & 0.39 & 0.25 & 0.2 & 6546 \\
\hline $\mathrm{Pd}, \mathrm{nPa}$ & 0.06 & 0.05 & 0.05 & 0.04 & 1394 & & & & & & 0.05 & 0.04 & 0.05 & 0.02 & 6021 \\
\hline Ma & 14.9 & 14.2 & 5.7 & 12 & 1394 & & & & & & 19.9 & 18.2 & 9.3 & 16 & 5968 \\
\hline Beta & 0.64 & 0.51 & 0.57 & 0.43 & 1394 & & & & & & 0.5 & 0.31 & 0.81 & 0.07 & 5968 \\
\hline & & & All (2496 & & & & & & & & & & $11(1123$ & & \\
\hline & Mean & Med & StDev & Mode & $\mathrm{n}$ & & & & & & Mean & Med & StDev & Mode & $\mathrm{n}$ \\
\hline $\mathrm{B}, \mathrm{nT}$ & 1.27 & 1.13 & 0.85 & 0.4 & 2488 & & & & & & 0.89 & 0.64 & 0.76 & 0.2 & 10705 \\
\hline $\mathrm{Pd}, \mathrm{nPa}$ & 0.13 & 0.09 & 0.13 & 0.03 & 2466 & & & & & & 0.1 & 0.06 & 0.12 & 0.03 & 9747 \\
\hline $\mathrm{Ma}$ & 11.6 & 10.5 & 5.7 & 8 & 2466 & & & & & & 15.7 & 13.5 & 9.2 & 8 & 9638 \\
\hline Beta & 0.54 & 0.39 & 0.57 & 0.13 & 2466 & & & & & & 0.46 & 0.27 & 0.8 & 0.07 & 9638 \\
\hline
\end{tabular}

${ }^{\mathrm{a}}$ Top panels provide values for Pioneer 10 and 11, which encountered Jupiter near solar minimum. Middle panels present Voyager 1 and 2 statistics near solar maximum. Bottom panels show the Ulysses and cumulative statistics.

summed distribution is observed in both the solar minimum and solar maximum subsets of the solar wind dynamic pressure data. The moments of the individual distributions, and their relative fractions, are similar to the values given in
Table 3 for the quiet and disturbed subsets at the solar cycle extrema.

[29] With a lognormal distribution, the mean of the underlying parameter $\left(\mathrm{P}_{\mathrm{d}}[\mathrm{nPa}]\right)$ is $\mathrm{e}^{\left(\mu+\sigma^{2} / 2\right)}$ and the median 

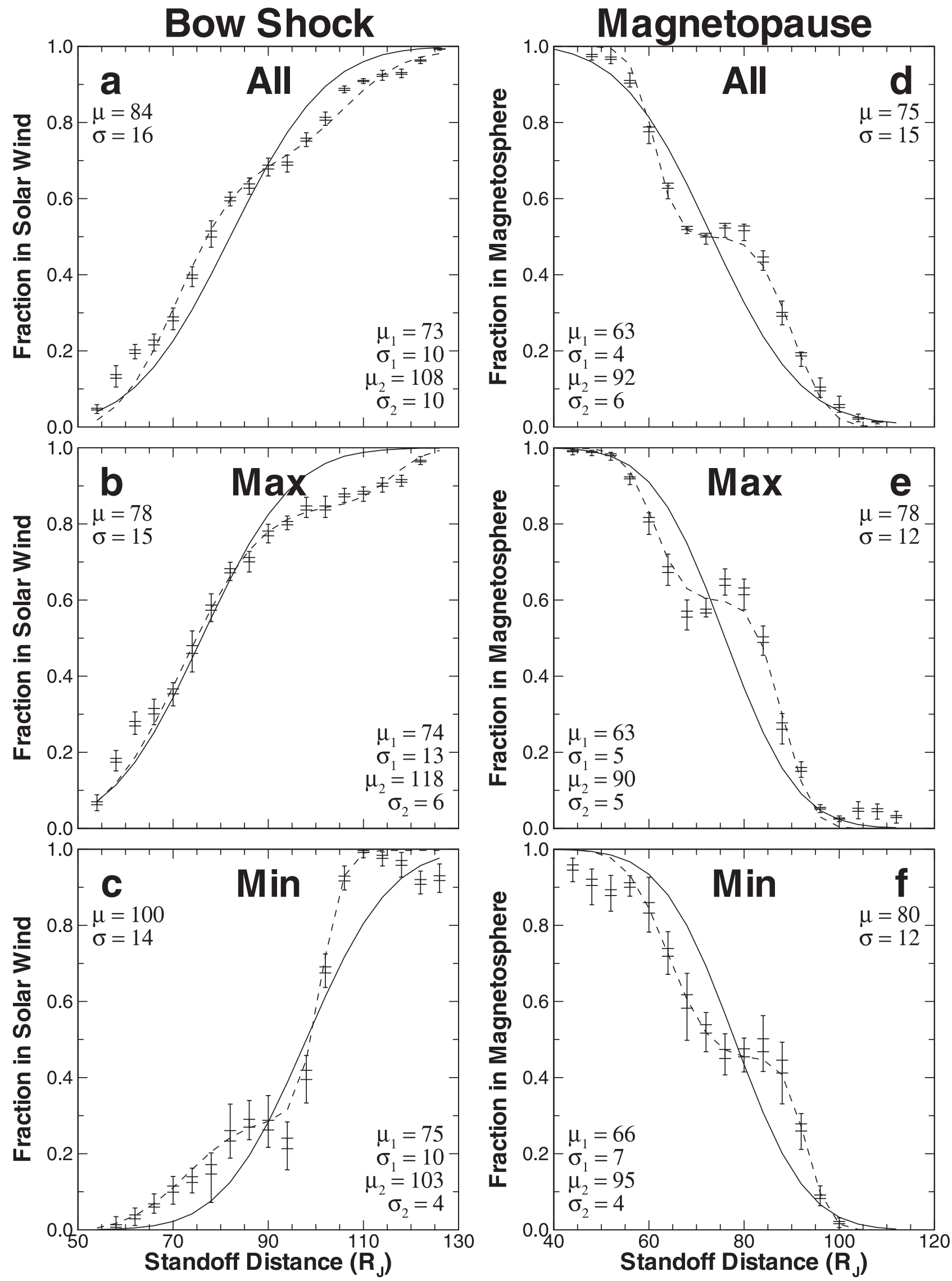
is $\mathrm{e}^{\mu}$. There is no direct way to get at the variance in the underlying parameter from a lognormal distribution. For the distribution observed, the mean pressures are $\mathrm{P}_{\mathrm{d} 1}=0.200$ $\mathrm{nPa}$ and $\mathrm{P}_{\mathrm{d} 2}=0.047 \mathrm{nPa}$ where the high pressure distribution is observed forty-two percent of the time. These values can be compared with the pressures required to generate the mean compressed $(0.306 \mathrm{nPa})$ and expanded $(0.039 \mathrm{nPa})$ state locations of the magnetopause from our polynomial fit (see section 4). The implication is that the dynamic pressure must increase by a factor of 7.8 to move the magnetopause from its expanded state to its compressed state. The observed solar wind dynamic pressure change between the two peaks in the distribution is only a factor of 4.3 . The observed separation in the preferred locations of the magnetopause cannot be reproduced by simply converting solar wind dynamic pressure into a standoff distance by using a power law expression that balances solar wind dynamic pressure against internal magnetic pressure at the boundary as was done by Slavin et al. [1985]. At Jupiter, the internal pressure at the magnetopause is maintained by a combination of magnetic, thermal plasma, and dynamic pressure components. In order to reproduce the large change in the magnetopause standoff distance, there must be changes in the contribution of internal plasma pressure components with a dependence on the external conditions that is different from that of the internal magnetic pressure.

[30] The data used to locate the bow shock and magnetopause positions in this study were separated into solar minimum and solar maximum components to identify solar cycle variations in the boundary locations or location variability. Galileo data from the Jupiter approach period in 1995 and the Pioneer results formed a solar minimum data set. Galileo observations from March 2000 to February 2001 and the Voyager observations formed a solar maximum data set. Figure 8 shows the cumulative probability distributions for the bow shock and magnetopause standoff distances at solar minimum, maximum, and for all observations. The bimodal magnetopause standoff distance distribution remains a persistent feature for all solar cycle conditions and the solar cycle variations appear to be small. The primary differences in the probability distributions are in the weighting factor required for each component in the bimodal distribution. At solar minimum, the compressed distribution is observed $55 \%$ of the time while at maximum it is observed only $40 \%$ of the time. This result is counterintuitive given the relatively higher fraction of disturbed, high-pressure solar wind at solar maximum. On the other hand, the compressed distribution is more compressed and the expanded distribution is less expanded at solar maximum than at solar minimum, which is consistent with expectation.

[31] The bow shock distribution is much more strongly affected by solar cycle variations. When all data are considered, the distribution is reasonably well characterized by a single, distribution with a mean standoff distance at 75 $\mathrm{R}_{\mathrm{J}}$. A poorly developed bimodal distribution is apparent with dominant $(70 \%)$ peak at $75 \mathrm{R}_{\mathrm{J}}$ and a weaker $(30 \%)$ peak at $105 \mathrm{R}_{\mathrm{J}}$. At solar maximum, the occurrence rate of the dominant distribution is as high as $85 \%$. At solar minimum, a statistically significant bimodal distribution develops in the data. The dominant distribution found in the complete data set remains present but at a much lower occurrence rate $(30 \%)$ while the distribution around $105 \mathrm{R}_{\mathrm{J}}$ occurs $70 \%$ of the time. The higher occurrence rate of the expanded distribution is consistent with the observation of a larger fraction of quiet solar wind at solar minimum. For most of the spacecraft flybys, and in the Galileo observations to date, there are many more observed bow shock crossings than magnetopause crossings. This is evidence that the bow shock moves more freely in response to changing solar wind and IMF conditions than does the magnetopause, as it does at Earth [Fairfield, 1971]. It is possible that under average and solar maximum conditions, the rapid motion of the bow shock smears the distribution function out over a large range of possible standoff distances. However, when the solar wind has long quiet periods during solar minimum, the expanded shock position may become more stable allowing the bimodal distribution to be resolved.

[32] The reduced number of points in each bin decreases the statistical confidence in the results when the data are separated into solar minimum and maximum subsets. This is particularly evident in the larger errors bars associated with the solar cycle extrema subsets. Both the bin ranges and the errors of the mean are larger in these subsets than when the entire data set is analyzed. However, the fitted magnetopause bimodal distribution functions for both the solar minimum and maximum subsets are statistically significant at greater than $99 \%$ confidence when analyzed by both the K-S test (overall data fit) and the F-test (improvement over simple Gaussian distribution fit) methods. When the bow shock data are analyzed in a similar manner, the single Gaussian distribution function is preferred at solar maximum and the bimodal distribution is favored at solar minimum.

\section{Summary and Discussion}

[33] We have developed new magnetopause and bow shock models using an approach that may prove applicable not only to Jupiter but also to other planets where observational data are sparse. Rather than simply identifying boundary crossings and determining a least squares fit to the events, we have described the boundaries in terms of the probability of being inside or outside boundary surfaces. We have used Jupiter MHD simulation results to define the boundary shapes and to determine how they vary in response to solar wind dynamic pressure. We then used the complete set of observations from all of the spacecraft that have encountered Jupiter in order to determine most probable boundary surface locations.

[34] Although the boundary shape models we have presented are described as functions of solar wind dynamic

Figure 8. (opposite) Bow shock and magnetopause (columns) fraction of observations outside (BS) and inside (MP) the boundary surfaces for all observations (top) and solar maximum (middle) and minimum (bottom) subsets of the observations. Solid lines are the single distribution function fits and the dashed lines are the bimodal distribution fits. Error bars and moments are derived in the same manner as in Figures $5 \mathrm{a}$ and $5 \mathrm{~d}$. 
pressure, they should not be used to infer the properties of the ambient solar wind with simple power law mappings. Our shape models of the equilibrium magnetopause position have a plausible dependence on solar wind dynamic pressure, as Table 1 shows, but additional factors (i.e. IMF Bz) influence the shape and the location of the system boundaries. In particular, our models are based on simulation results where the IMF is vanishing. However, as Table 1 also shows, the magnetopause shapes at small values of IMF $B_{z}( \pm 0.42 \mathrm{nT})$ and $P_{d}=0.090 \mathrm{nPa}$ are very similar to the shape at $\mathrm{B}_{\mathrm{z}}=0.0 \mathrm{nT}$ and $\mathrm{P}_{\mathrm{d}}=0.045 \mathrm{nPa}$. In other words, the boundary shapes that are found at low values of IMF $B_{z}$ are well described by our families of bow shock and magnetopause surfaces. Lastly, the models presented here are derived from the locations of the boundaries under steady-state conditions. Under dynamic solar wind conditions, the boundary shapes are likely to become distorted as they respond to changes in the solar wind and IMF.

[35] The simulation results from which our boundary surface shape models are derived are limited in size and spatial resolution. The bow shock surface exits the sides and top of the simulation box in the near tail region less than $150 \mathrm{R}_{\mathrm{J}}$ downstream of the planet. Our bow shock shape models based on the shapes inferred from the simulations should not be extended beyond about $150 \mathrm{R}_{\mathrm{J}}$ downstream. The magnetopause surface does not exit the simulation box (except out the back at $450 \mathrm{R}_{\mathrm{J}}$ ) but is not well fit by our models beyond $200-250 \mathrm{R}_{\mathrm{J}}$ downstream. In order to fit the distant magnetopause, a different functional form is required. In the north-south dimension the model is not a good representation of the magnetopause at values of $\mathrm{Z}$ $>100 \mathrm{R}_{\mathrm{J}}$. In order to obtain a good fit to the simulation in the equatorial plane, data near the equatorial plane were more heavily weighted than those at large values of $\mathrm{Z}$. There are additional shape considerations at high latitude associated with the cusp region that are not well represented by this functional form. There are no observations of the Jovian system in the regions at high values of $\mathrm{Z}$ where our shape model breaks down so these deficiencies have not affected our statistics.

[36] We have found that the magnetopause at Jupiter has two preferred locations, one representing a compressed magnetosphere and the other an expanded magnetosphere. Differences in the solar wind within and between disturbed regions are presumably responsible for setting up this bimodal distribution. In particular, the bimodal distribution observed in the solar wind dynamic pressure is likely to contribute to the tendency of the magnetopause to have two preferred locations, but internal pressure changes are also required. Variations associated with solar cycle differences in the magnetopause location have been investigated but the effect is small. The bimodal distribution is present, and nearly constant, throughout the solar cycle. The magnetosphere appears to be slightly more compressed at solar maximum than at solar minimum. The bow shock location distribution can also be described as bimodal but this description is not statistically rigorous. The speed with which the bow shock can adjust to solar wind conditions tends to smear out the distribution. At solar maximum, there are few observations of an expanded shock location. The distribution is well described by a single Gaussian distribution function with a standoff distance of about $78 \mathrm{R}_{\mathrm{J}}$ that is consistent with the compressed state location in the complete data set. At solar minimum, a bimodal distribution in the shock location is clearly observed. The compressed state location still persists but the expanded state location (near $105 \mathrm{R}_{\mathrm{J}}$ ) that is hinted at in the complete data set becomes fully resolved.

[37] Acknowledgments. The authors wish to extend great thanks to Joseph Mafi who created all of the figures in this manuscript. Without Joe's help our results would be much less clearly presented. We would also like to thank Dr. Paul O'Brien who provided many useful comments during discussions of sampling biases within our data and statistical significance testing of our models. Spacecraft trajectory data used to define the fraction of time inside and outside of boundary surfaces were derived from SPICE kernels obtained from the NAIF node of the Planetary Data System. The data used to characterize the statistical properties of the solar wind near Jupiter were acquired from COHOWeb, an NSSDC resource. The work at Nagoya University was supported by grants in aid from the Ministry of Education, Science and Culture. Computing support was provided by the Computer Center of Nagoya University and by the National Partnership for Advanced Computing and Infrastructure (NPACI). NASA directly funded this research through contract JPL 958694 and NASA grant NAG5-10282. UCLA Institute of Geophysics and Planetary Physics Publication Number 5704 .

\section{References}

Bame, S. J., B. L. Barraclough, W. C. Feldman, G. R. Gisler, J. T. Gosling, D. J. McComas, J. L. Philips, M. F. Thomsen, B. E. Goldstein, and M. Neugebauer, Jupiter's magnetosphere: Plasma description from the Ulysses flyby, Science, 257, 1539, 1992.

Engel, I. M., and D. B. Beard, Idealized Jovian magnetopause shape and field, J. Geophys. Res., 85(A2), 579, 1980.

Fairfield, D. H., Average and unusual locations of the Earth's magnetopause and bow shock, J. Geophys. Res., 76(28), 6700, 1971.

Farris, M. H., and C. T. Russell, Determining the standoff distance of the bow shock: Mach number dependence and use of models, J. Geophys. Res., 99, 17,681, 1994.

Gazis, P. R., Solar cycle variation in the heliosphere, Rev. Geophys, 34(3), 379,1996

Hill, T. W., and A. J. Dessler, Configuration of the Jovian magnetosphere, Geophys. Res. Lett., 1(1), 3, 1974.

Huddleston, D. E., C. T. Russell, M. G. Kivelson, K. K. Khurana, and L. Bennett, Location and shape of the Jovian magnetopause and bow shock, J. Geophys. Res., 103(E9), 20,075, 1998.

Intriligator, D. S., and J. H. Wolfe, Results of the plasma analyzer experiment on Pioneers 10 and 11, in Jupiter, edited by T. Geherls, pp. 848869, Univ. of Ariz. Press, Tucson, 1976.

Kivelson, M. G., et al., Galileo at Jupiter: Changing states of the magnetosphere and first looks at Io and Ganymede, Adv. Space Res., 20(2), 193, 1997.

Kivelson, M. G., K. K. Khurana, and R. J. Walker, Sheared magnetic field structure in Jupiter's dusk magnetosphere: Implications for return currents, J. Geophys. Res., 107(A7), 1116, doi:10.1029/2001JA000251, 2002.

Lepping, R. P., L. F. Burlaga, and L. W. Klein, Jupiter's magnetopause, bow shock, and 10-hour modulated magnetosheath: Voyagers 1 and 2, Geophys. Res. Lett., 8(1), 99, 1981a.

Lepping, R. P., M. J. Silverstein, and N. F. Ness, Magnetic field measurements at Jupiter by Voyagers 1 and 2: Daily plots of 48 second averages, NASA Tech., 83,864, 1981b.

Ogino, T., R. J. Walker, and M. G. Kivelson, A global magnetohydrodynamic simulation of the Jovian magnetosphere, J. Geophys. Res., 103, 225, 1998

Petrinec, S. M., and C. T. Russell, Hydrodynamic and MHD equations across the bow shock and along the surfaces of planetary obstacles, Space Sci. Rev., 79, 757, 1997

Richardson, J. D., J. W. Belcher, A. J. Lazarus, K. I. Paularena, and P. R. Gazis, Statistical properties of the solar wind, in Proceedings of the Eighth International Solar Wind Conference, Dana Point, CA, edited by D. Winterhalter et al., AIP Conf. Proc., 382, 483-486, 1996.

Riley, P., S. J. Bame, B. L. Barraclough, W. C. Feldman, J. T. Gosling, G. W. Hoogeveen, D. J. McComas, B. E. Goldstein, and M. Neugebauer, ULYSSES solar wind plasma observations at high latitudes, Adv. Space Res., 20, 15, 1997.

Slavin, J. A., R. E. Holzer, J. R. Spreiter, and S. S. Stahara, Planetary Mach cones: Theory and observation, J. Geophys. Res., 89(A5), 2708, 1984.

Slavin, J. A., E. J. Smith, J. R. Spreiter, and S. S. Stahara, Solar wind flow 
about the outer planets: Gas dynamic modeling of the Jupiter and Saturn bow shocks, J. Geophys. Res., 90(A7), 6275, 1985.

Slavin, J. A., G. Jungman, and E. J. Smith, The interplanetary magnetic field during solar cycle 21: ISEE_3/ICE observations, Geophys. Res. Lett., 13(6), 513, 1986.

Smith, E. J., and J. H. Wolfe, Fields and plasmas in the outer solar system, Space Sci. Rev., 23, 217, 1979.

Smith, E. J., R. W. Fillilius, and J. H. Wolfe, Compression of Jupiter's magnetosphere by the solar wind, J. Geophys Res., 83, 4733, 1978.

Smith, Z. K., M. Dryer, R. W. Fillius, E. J. Smith, and J. H. Wolfe, Compression of Jupiter's magnetosphere by the solar wind: Reexamination via MHD simulation of evolving corotating interaction regions, J. Geophys. Res., 86(A8), 6773, 1981.

Spreiter, J. R., and S. S. Stahara, Magnetohydrodynamic and gasdynamic theories for planetary bow waves, in Collisionless Shocks in the Heliosphere: A Review of Current Research, Geophys. Monogr. Ser., vol. 35, edited by B. Tsurutani and T. R. G. Stone, pp. 85-107, AGU, Washington, D. C., 1985.

Spreiter, J. R., A. L. Summers, and A. Y. Alksne, Hydromagnetic flow around the magnetosphere, Planet. Space. Sci., 14, 223, 1966.
Stahara, S. S., R. R. Rachiele, J. R. Spreiter, and J. A. Slavin, A three dimensional gasdynamic model for the solar wind flow past nonaxisymmetric magnetospheres: Application to Jupiter and Saturn, J. Geophys. Res., 94(A10), 13,353, 1989.

Walker, R. J., T. Ogino, and M. G. Kivelson, Magnetohydrodynamic simulations of the effects of the solar wind on the Jovian magnetosphere, Planet. Space Sci, 49, 237-245, 2001.

Webb, D. F., and R. A. Howard, The solar cycle variation of coronal mass ejections and the solar wind mass flux, J. Geophys. Res., 99(A3), 4201, 1994.

Winterhalter, D., E. J. Smith, J. H. Wolfe, and J. A. Slavin, Spatial gradients in the heliospheric magnetic field: Pioneer 11 observations between $1 \mathrm{AU}$ and 24 AU over solar cycle 21, J. Geophys. Res., 95(A1), 1, 1990.

S. P. Joy, K. K. Khurana, M. G. Kivelson, C. T. Russell, and R. J. Walker, Institute of Geophysics and Planetary Physics, University of California, Los Angeles, 6844 Slichter Hall, CA 90095-1567, USA.

T. Ogino, Solar Terrestrial Environment Laboratory, Nagoya University, Toyokawa, Aichi, Japan. 